\title{
Rapid Photoracemization of Chiral Alkyl Aryl Sulfoxides
}

Kosho Makino, ${ }^{1}$ Kumi Tozawa, ${ }^{1}$ Yuki Tanaka, ${ }^{1}$ Akiko Inagaki, ${ }^{2}$ Hidetsugu Tabata,${ }^{3}$ Tetsuta Oshitari, ${ }^{2}$ Hideaki Natsugari, ${ }^{3}$ Hideyo Takahashi*1

${ }^{1}$ Faculty of Pharmaceutical Sciences, Tokyo University of Science, 2641 Yamazaki, Noda-shi, Chiba 278-8510, Japan

${ }^{2}$ Department of Chemistry, Tokyo Metropolitan University, 1-1 Minami-Osawa, Hachioji, Tokyo 192-0397, Japan

${ }^{3}$ Faculty of Pharma Sciences, Teikyo University, 2-11-1 Kaga, Itabashi-ku, Tokyo 173-8605, Japan ${ }^{4}$ Graduate School of Pharmaceutical Science, The University of Tokyo, 7-3-1 Hongo, Bunkyo-ku, Tokyo 113-0033, Japan

hide-tak@rs.tus.ac.jp

\section{Contents}

1. Pseudo-first-order rate constants, $\boldsymbol{k}_{\mathrm{obs}}$, and second-order rate constants $\boldsymbol{k}_{2} \ldots \ldots \ldots \ldots . . . \mathrm{S2}$

2. Electrochemical measurements................................................S10

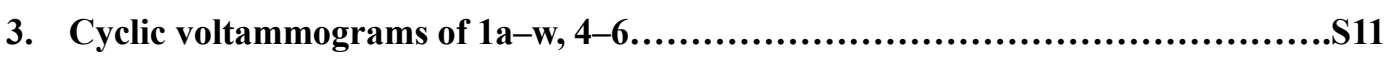

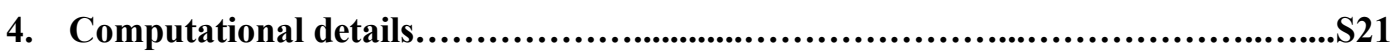

5. Chiral HPLC charts of 1a-w and their optical properties..........................S24

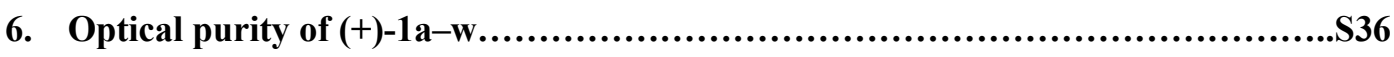

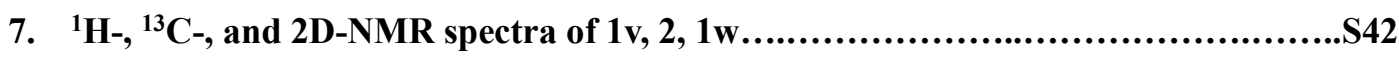




\section{Pseudo-first-order rate constants, $\boldsymbol{k}_{\mathrm{obs}}$, and second-order rate constants $\boldsymbol{k}_{2}$}

Table S1. Racemization rate $\left(k_{\mathrm{obs}}\right)$ of $(+)-\mathbf{1 p}(0.01 \mathrm{M})$ with $\mathrm{TPT}^{+}$at r.t. in $\mathrm{MeCN}$.

\begin{tabular}{ccc} 
& $\mathrm{TPT}^{+}(\mathrm{mol} \%)$ & $k_{\mathrm{obs}}\left(s^{-1}\right)$ \\
\cline { 2 - 3 } & 0.1 & $1.03 \times 10^{-3}$ \\
0.5 & $3.94 \times 10^{-3}$ \\
$1 \mathrm{p}$ & 0.75 & $8.90 \times 10^{-3}$ \\
1.25 & $1.13 \times 10^{-2}$ \\
\end{tabular}

Figure S1. First-order dependence of the rate of racemization on $\mathrm{TPT}^{+}$for the reaction of $(+)-\mathbf{1 p}$

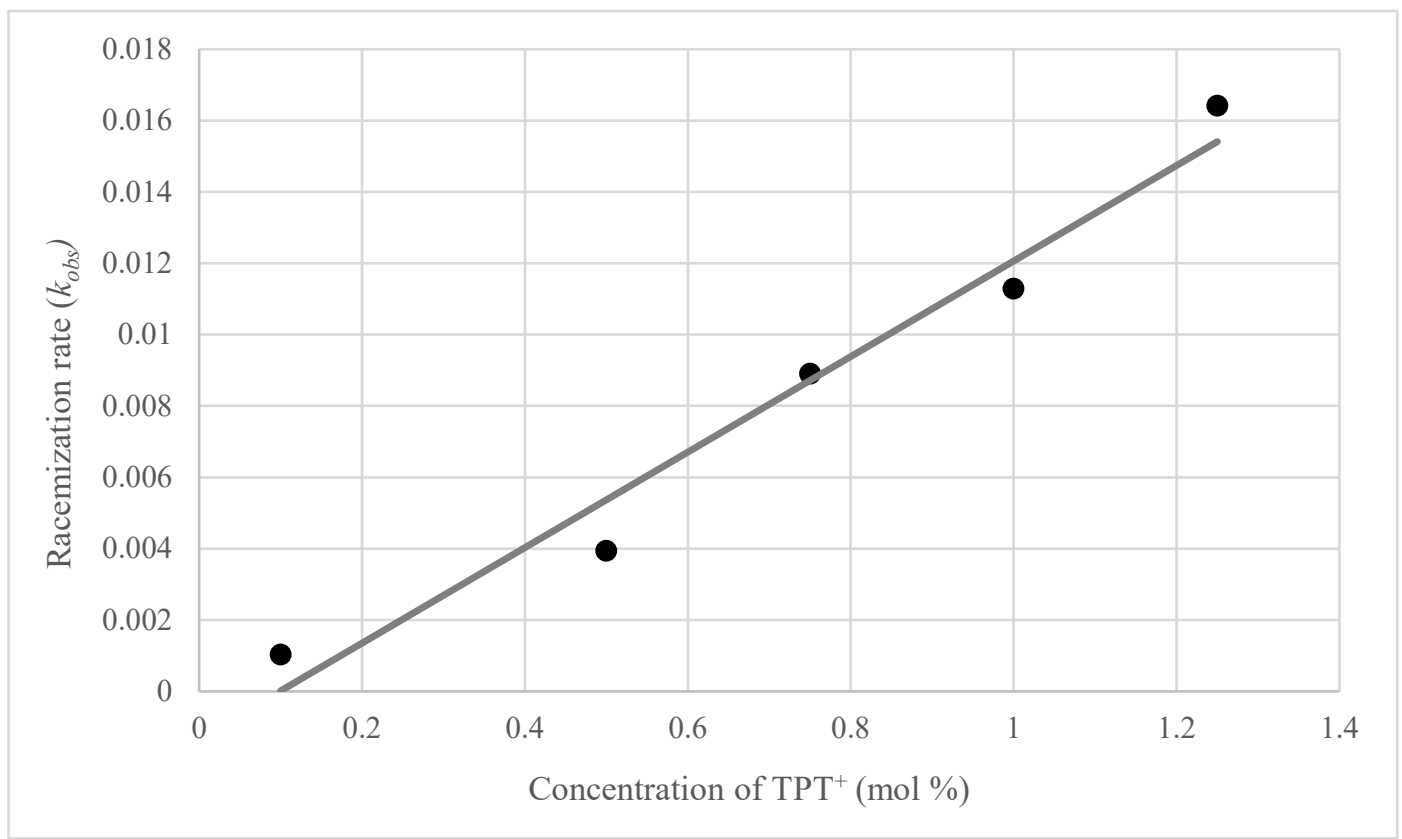

Racemization rates were shown to follow pseudo-first-order rate constants, $k_{\text {obs }}$ were determined from the slope of the straight line obtained when $-\ln \left(\% \mathrm{ee}_{\mathrm{t}} / \% \mathrm{ee}_{0}\right)$ is plotted against time.

$$
\begin{aligned}
-\ln \left(\% \mathrm{ee}_{\mathrm{t}} / \% \mathrm{ee}_{0}\right) & =k_{\text {obs }} \mathrm{t} \\
k_{\mathrm{obs}} & =k_{2}\left[\mathrm{TPT}^{+}\right]_{0} \\
t_{1 / 2} & =\ln (2) / k_{\mathrm{obs}}
\end{aligned}
$$



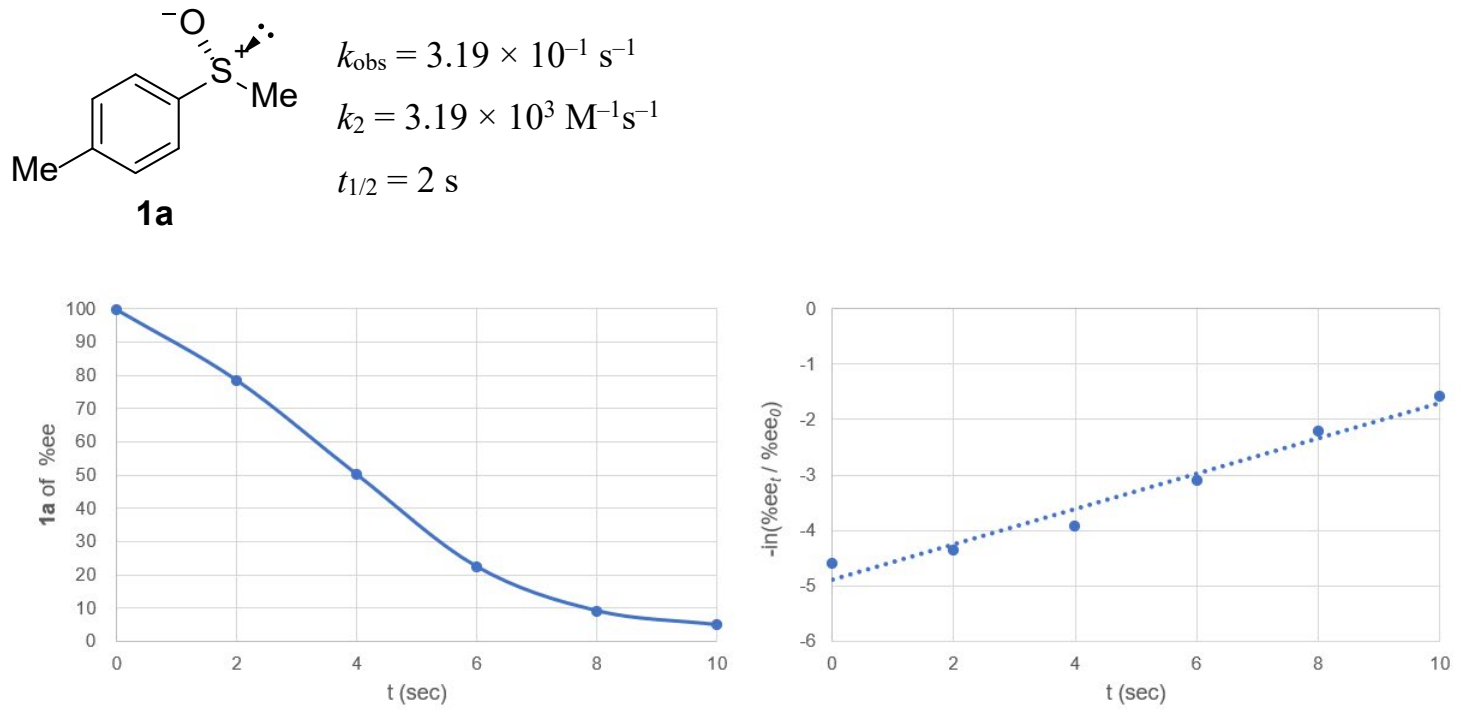<smiles>C[Sn](C)(O)c1ccccc1</smiles>

$$
\begin{aligned}
& k_{\text {obs }}=1.77 \mathrm{~s}^{-1} \\
& k_{2}=1.77 \times 10^{4} \mathrm{M}^{-1} \mathrm{~s}^{-1}
\end{aligned}
$$

$1 b$ $t_{1 / 2}=0.4 \mathrm{~s}$
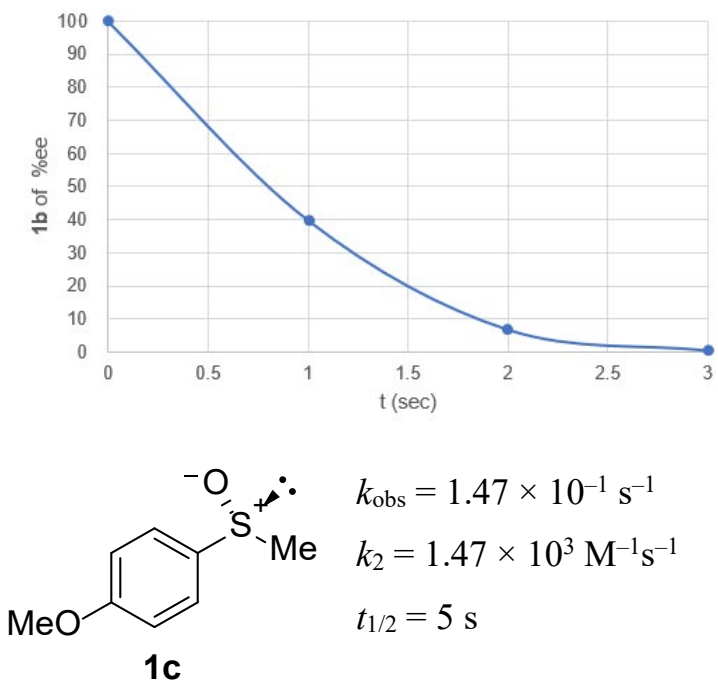

$$
\begin{aligned}
& k_{\mathrm{obs}}=1.47 \times 10^{-1} \mathrm{~s}^{-1} \\
& k_{2}=1.47 \times 10^{3} \mathrm{M}^{-1} \mathrm{~s}^{-1} \\
& t_{1 / 2}=5 \mathrm{~s}
\end{aligned}
$$

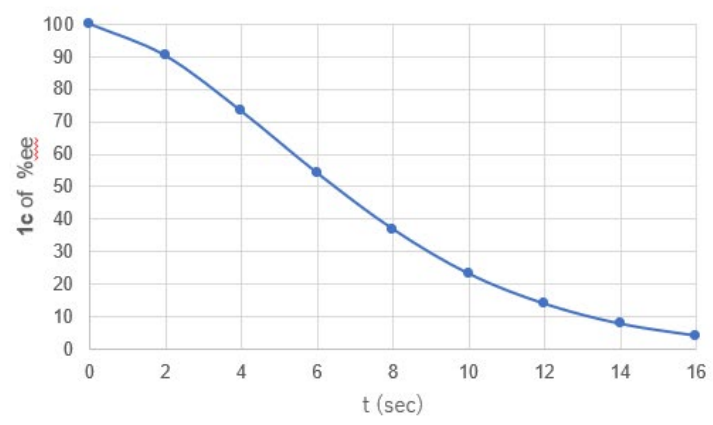

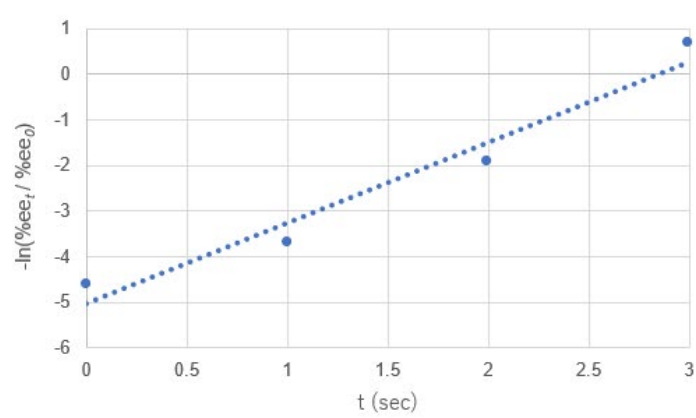

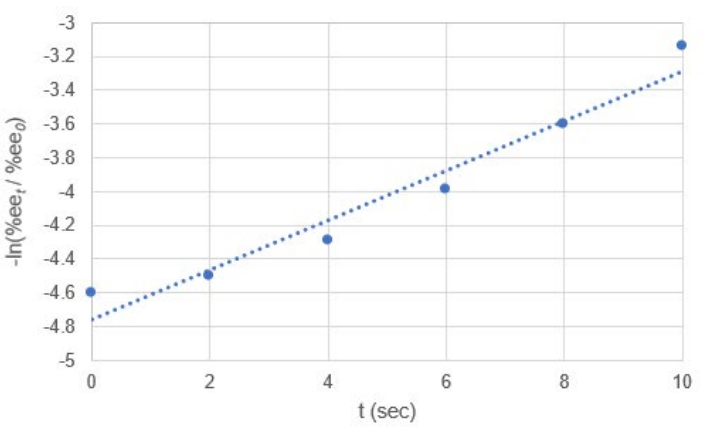

SB 

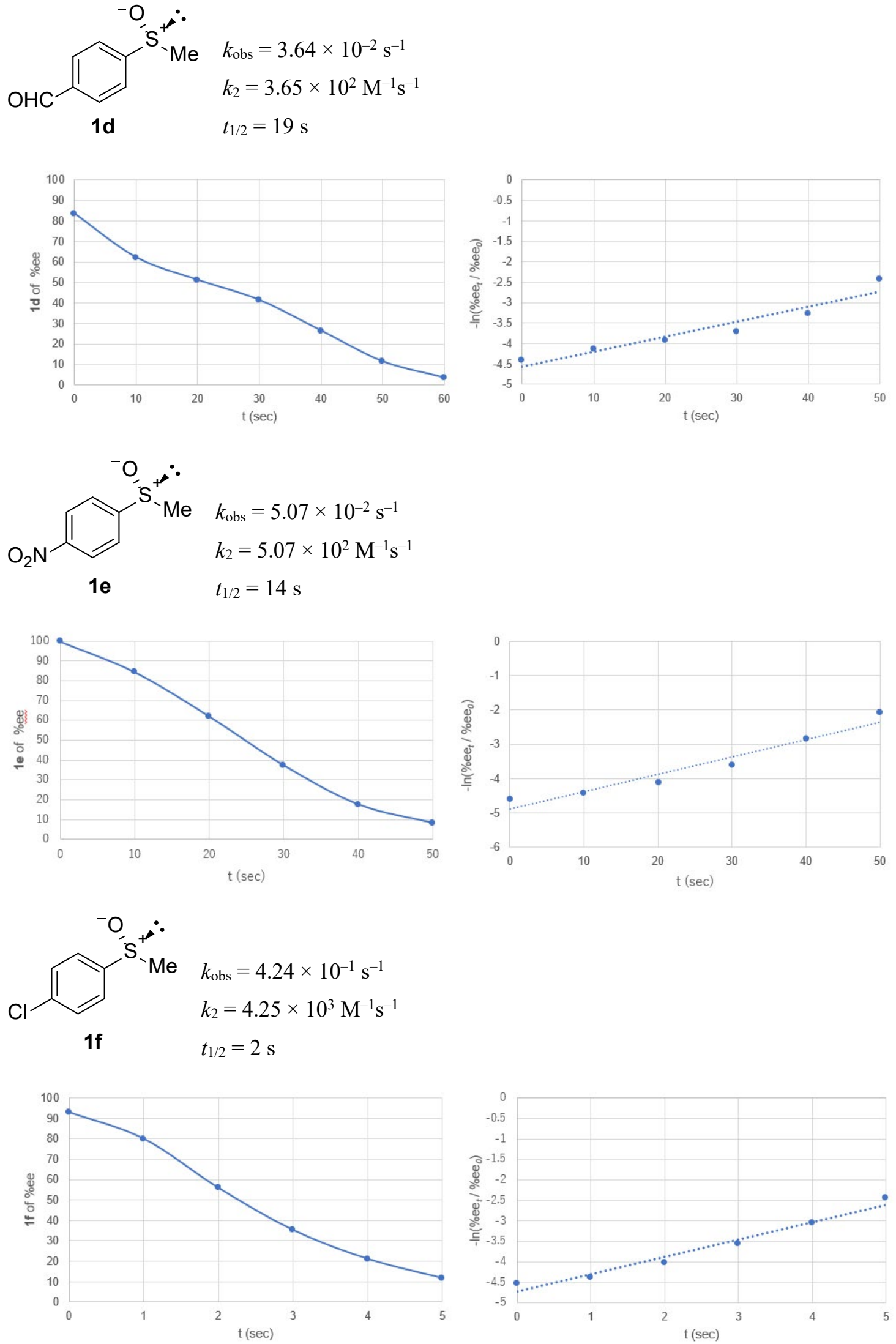
$\underbrace{}_{1 \mathrm{~g}} \begin{aligned} & k_{\mathrm{obs}}=3.78 \times 10^{-1} \mathrm{~s}^{-1} \\ & k_{1 / 2}=2 \mathrm{~s}\end{aligned}$
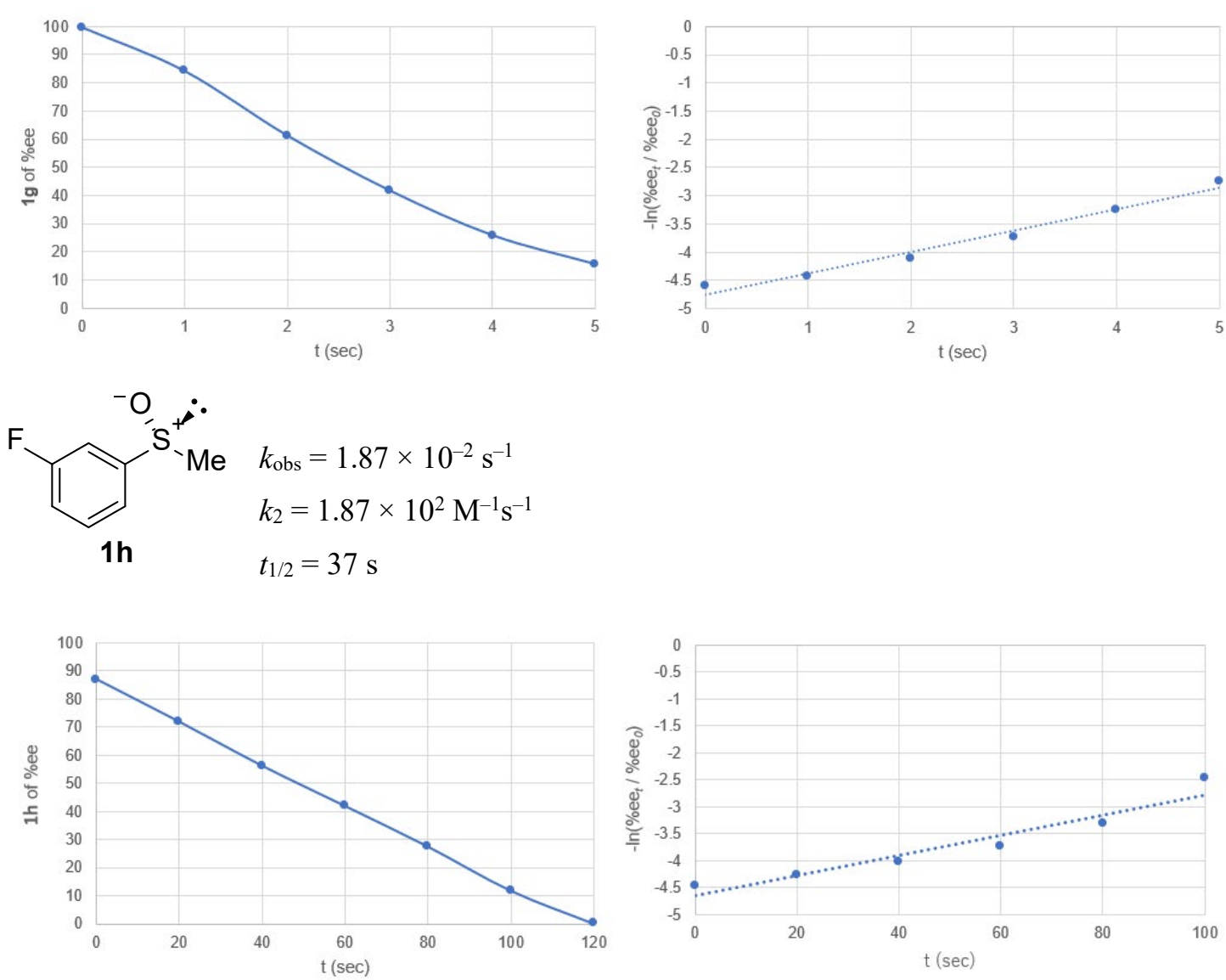
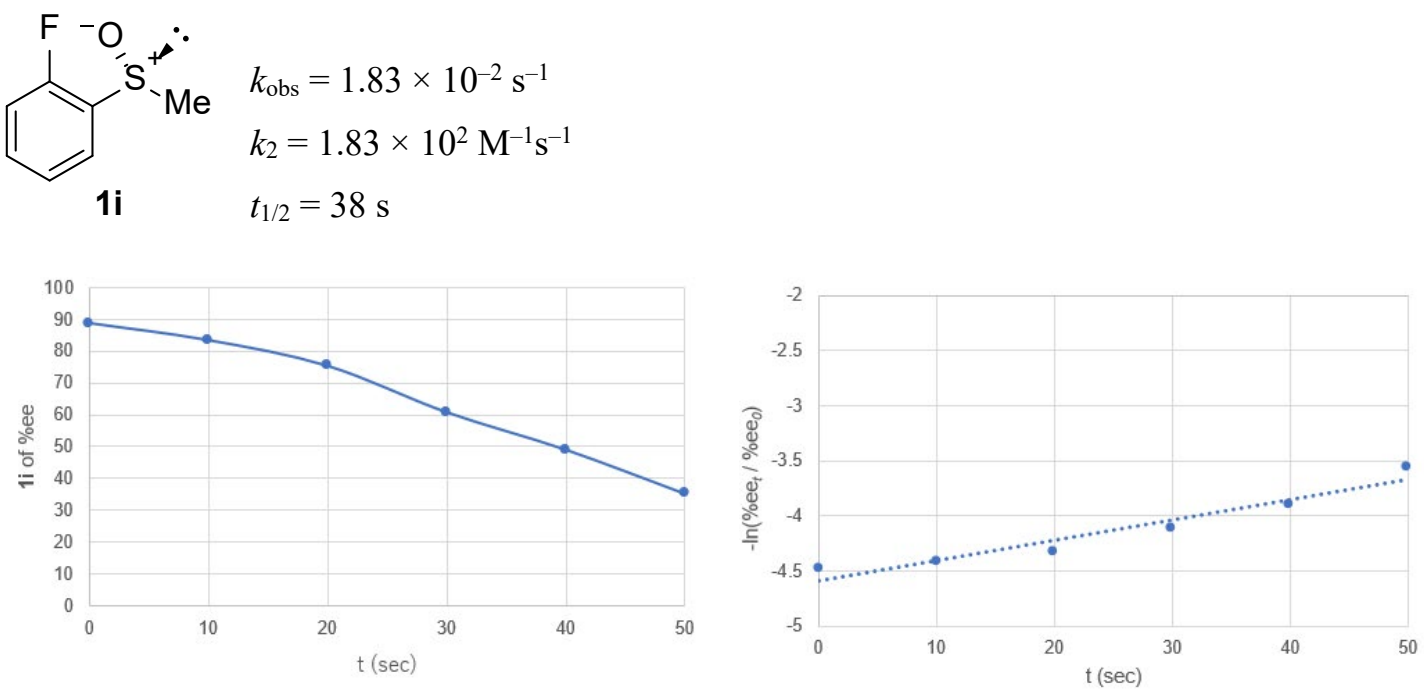

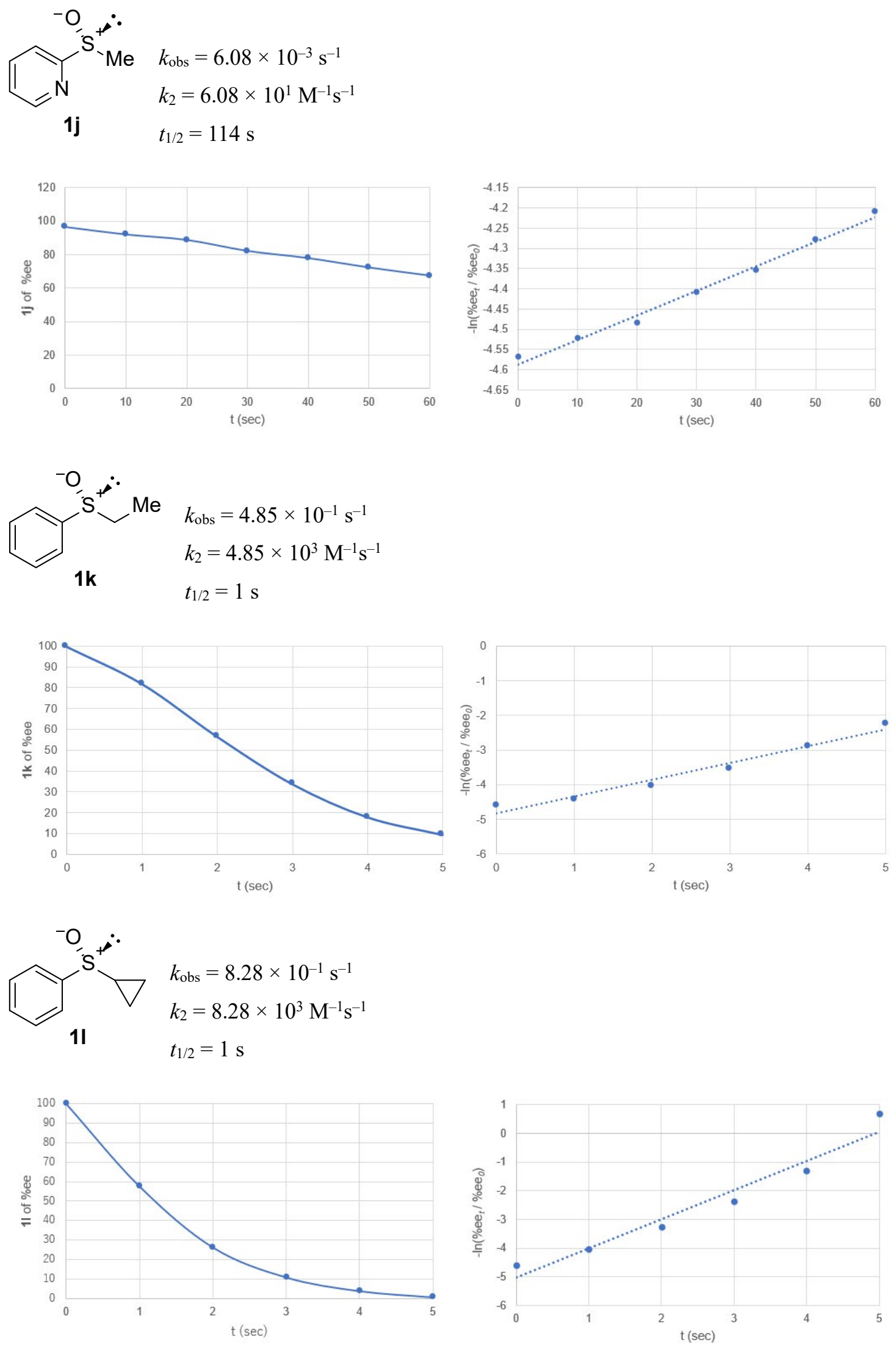

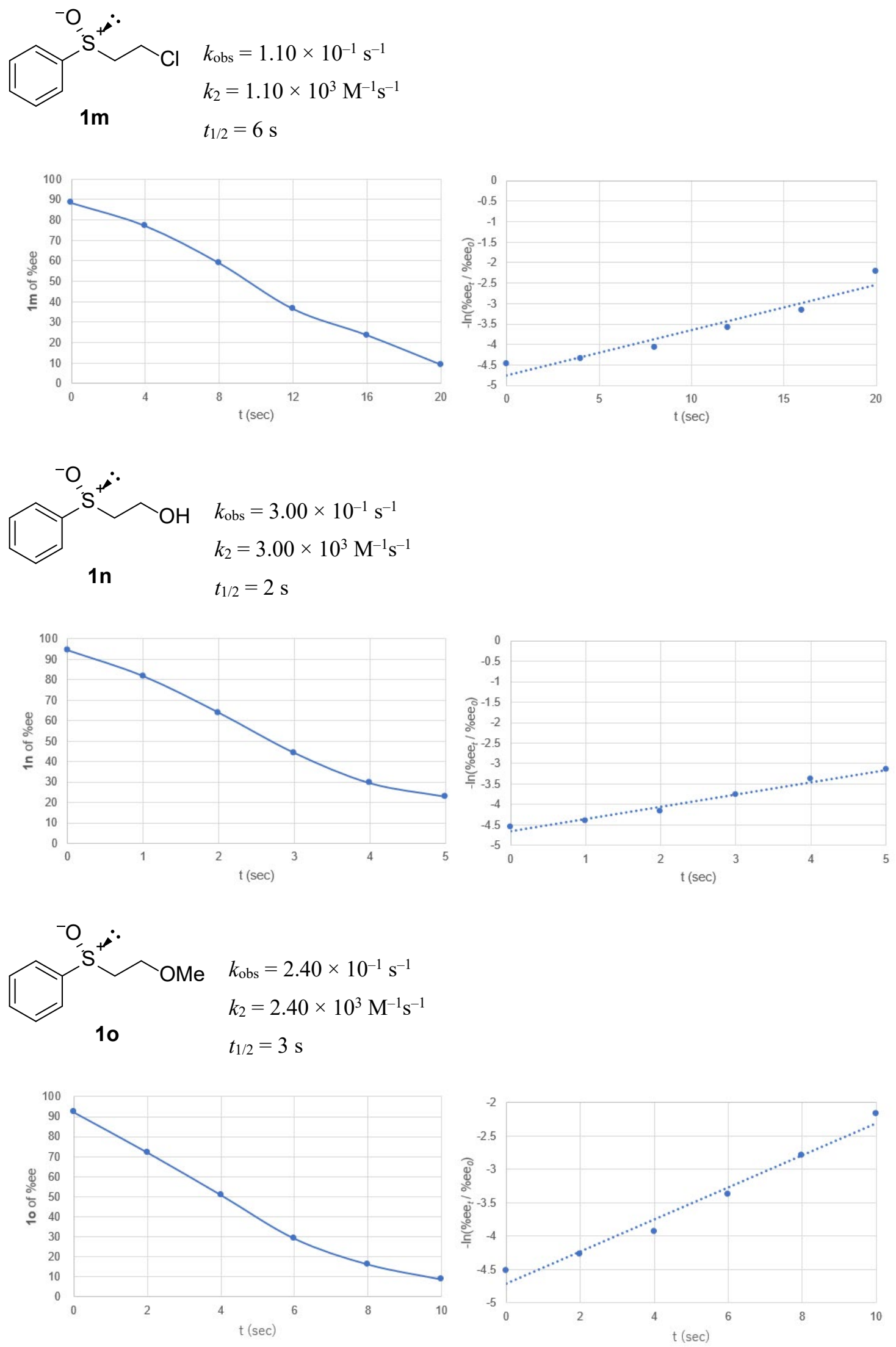

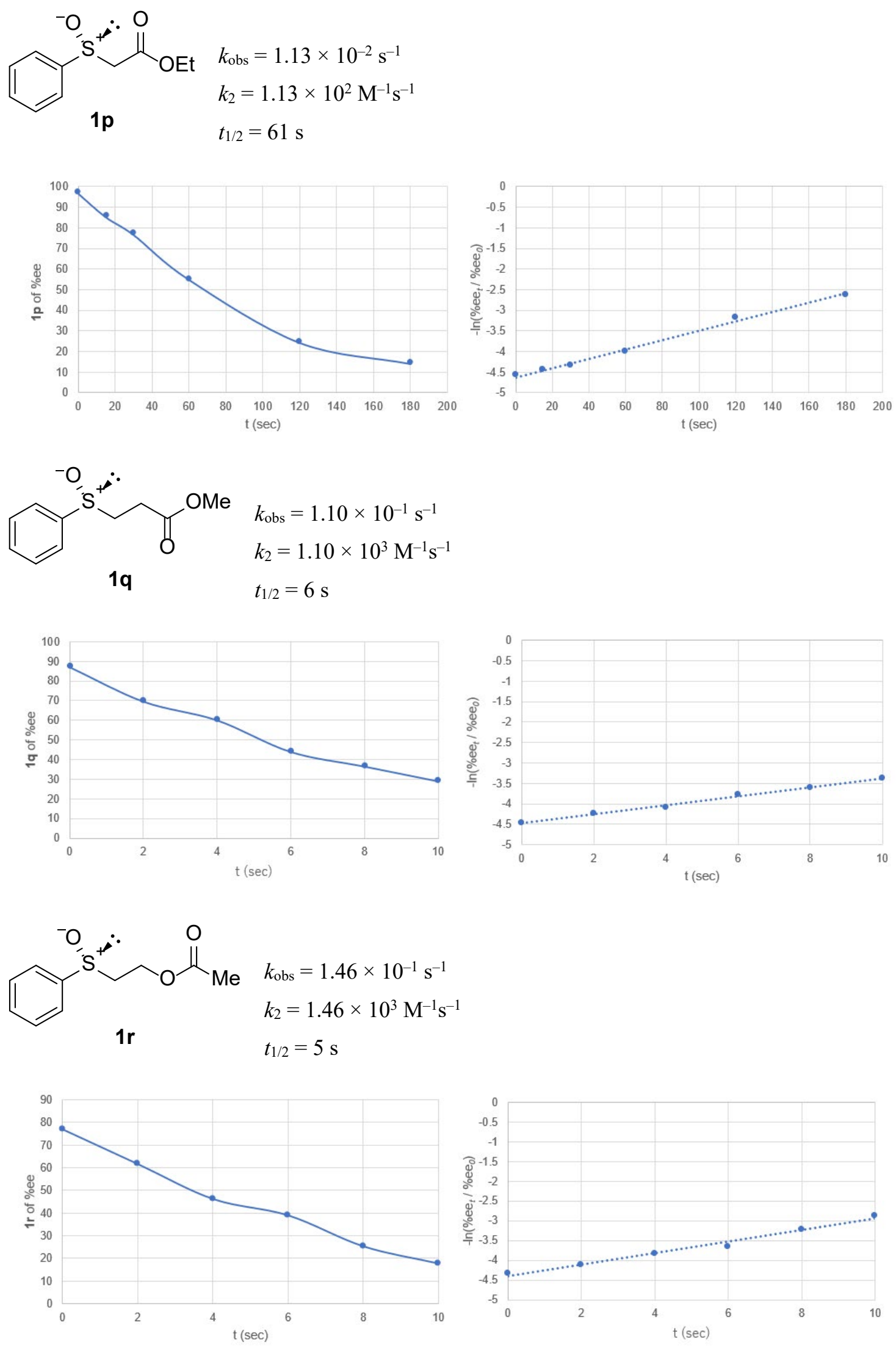

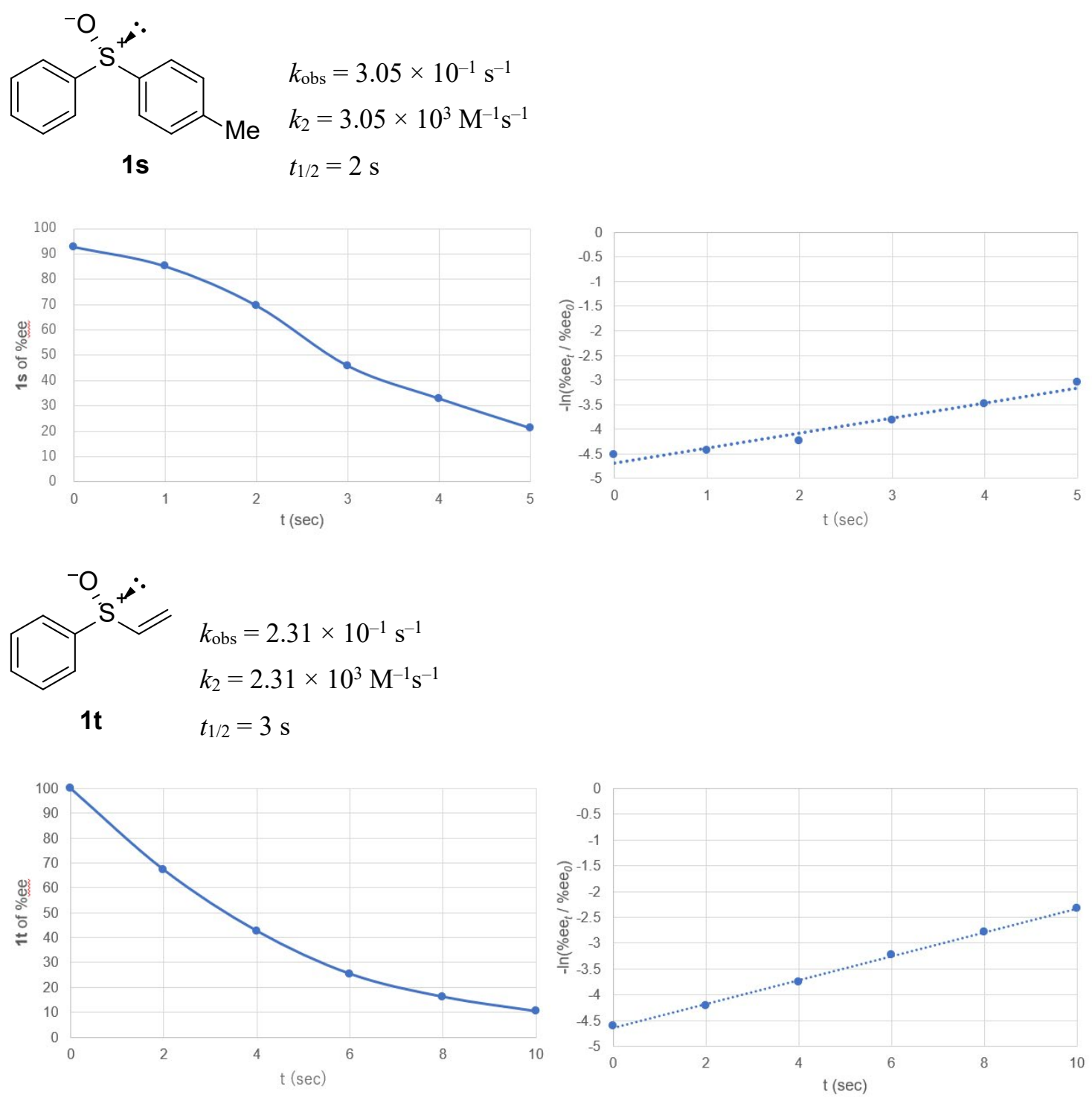


\section{Electrochemical measurements}

Electrochemical potentials were obtained with a standard set of conditions to main internal consistency. Cyclic voltammograms were collected with a computer-controlled potentiostat ECstat-301 (EC Frontier Co., Ltd). Samples were prepared with $0.021 \mathrm{mmol}$ of substrate in $7 \mathrm{~mL}$ of $0.1 \mathrm{M}$ tetra- $n$-butylammonium perchlorate in dry, degassed acetonitrile. Measurements employed a glassy carbon working electrode, platinum wire counterelectrode, $0.1 \mathrm{M} \mathrm{AgNO}_{3}$, $0.1 \mathrm{M}$ tetra- $n$-butylammonium perchlorate-silver nitrate reference electrode, and a scan rate of 25 $\mathrm{mV} / \mathrm{s}$. Reductions were measured by scanning potentials in the negative direction and oxidations in the positive direction; the glassy carbon electrode was polished between each scan. The ferrocene/ferrocenium couple $\left(\mathrm{Fc} / \mathrm{Fc}^{+}\right)$was also measured in the same electrochemical system, and the electrode potential was reported as values referred to the standard potential of the system. The reference value was converted to SCE by adding $0.447 \mathrm{~V}$ (in $\mathrm{CH}_{3} \mathrm{CN}$ ). 
3. Cyclic voltammograms of 1a-w, 4-6.
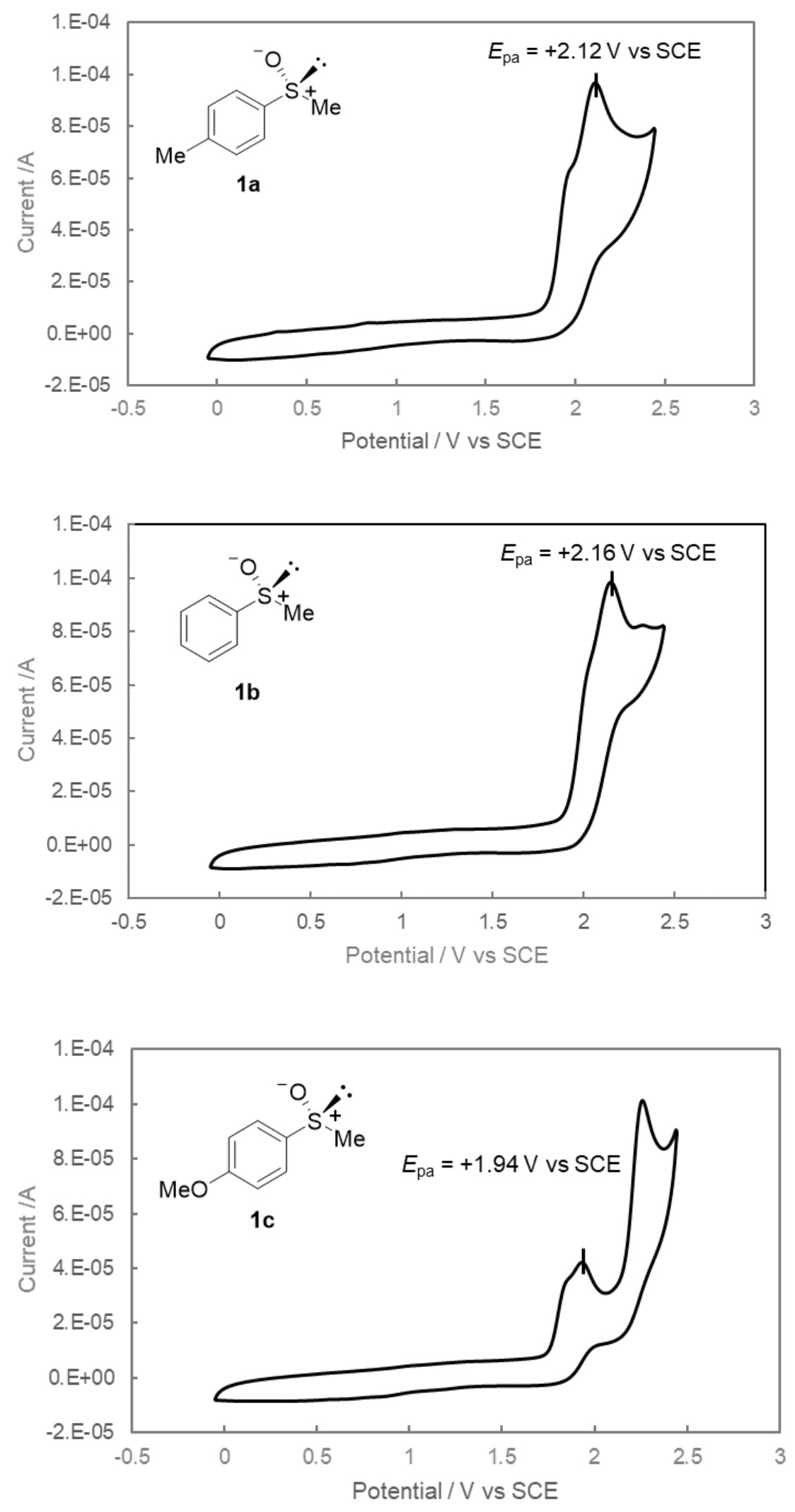

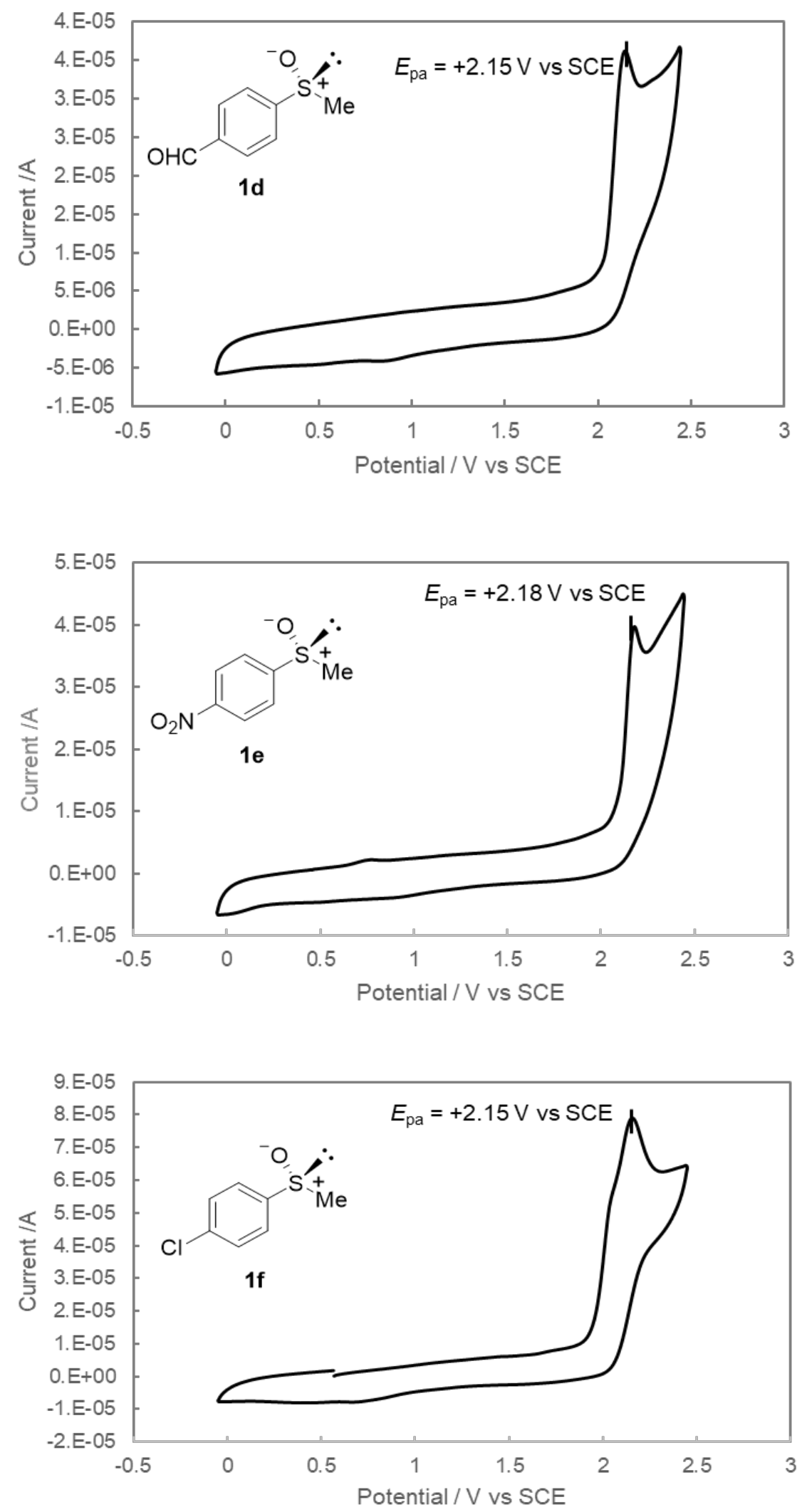

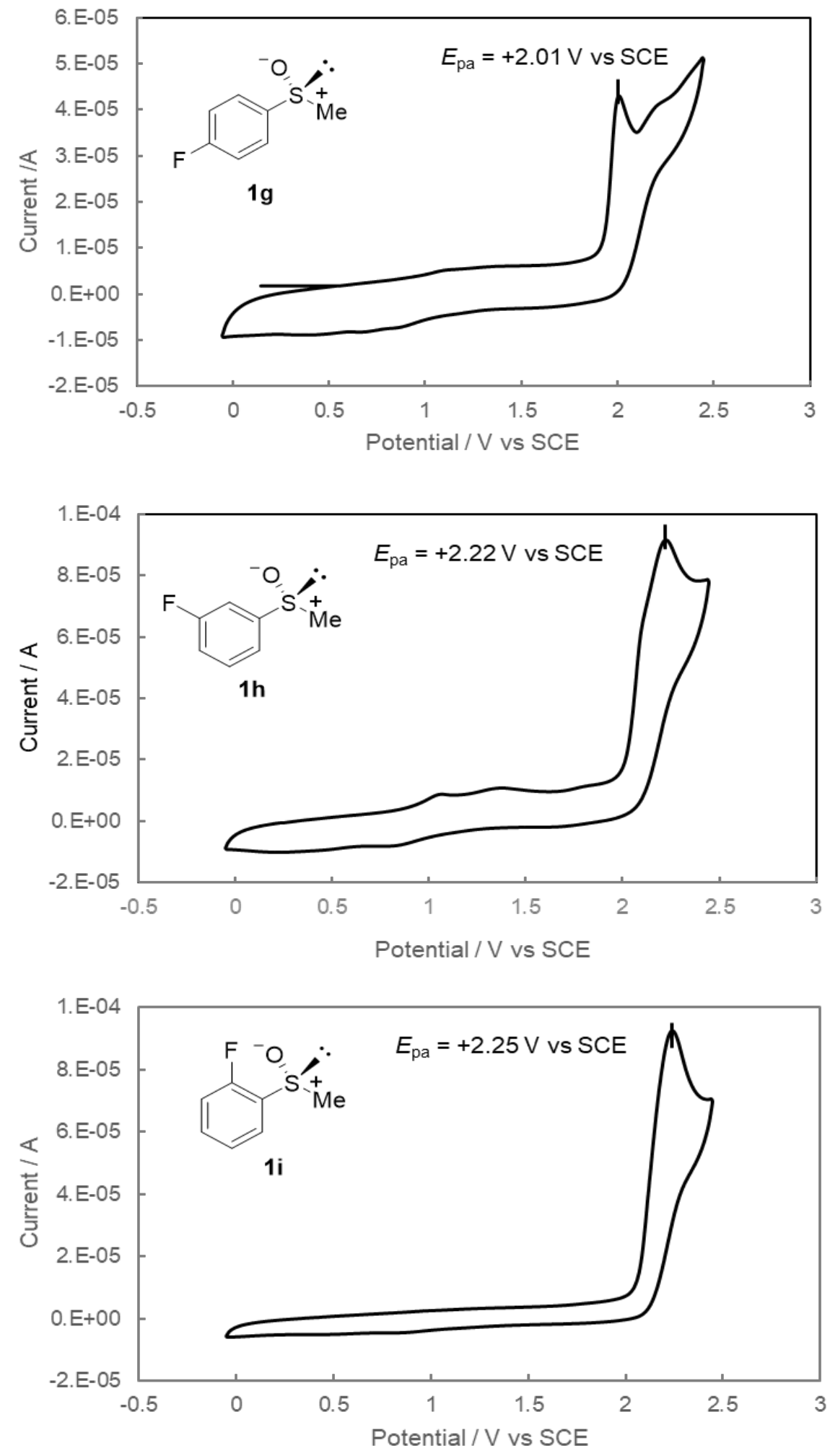

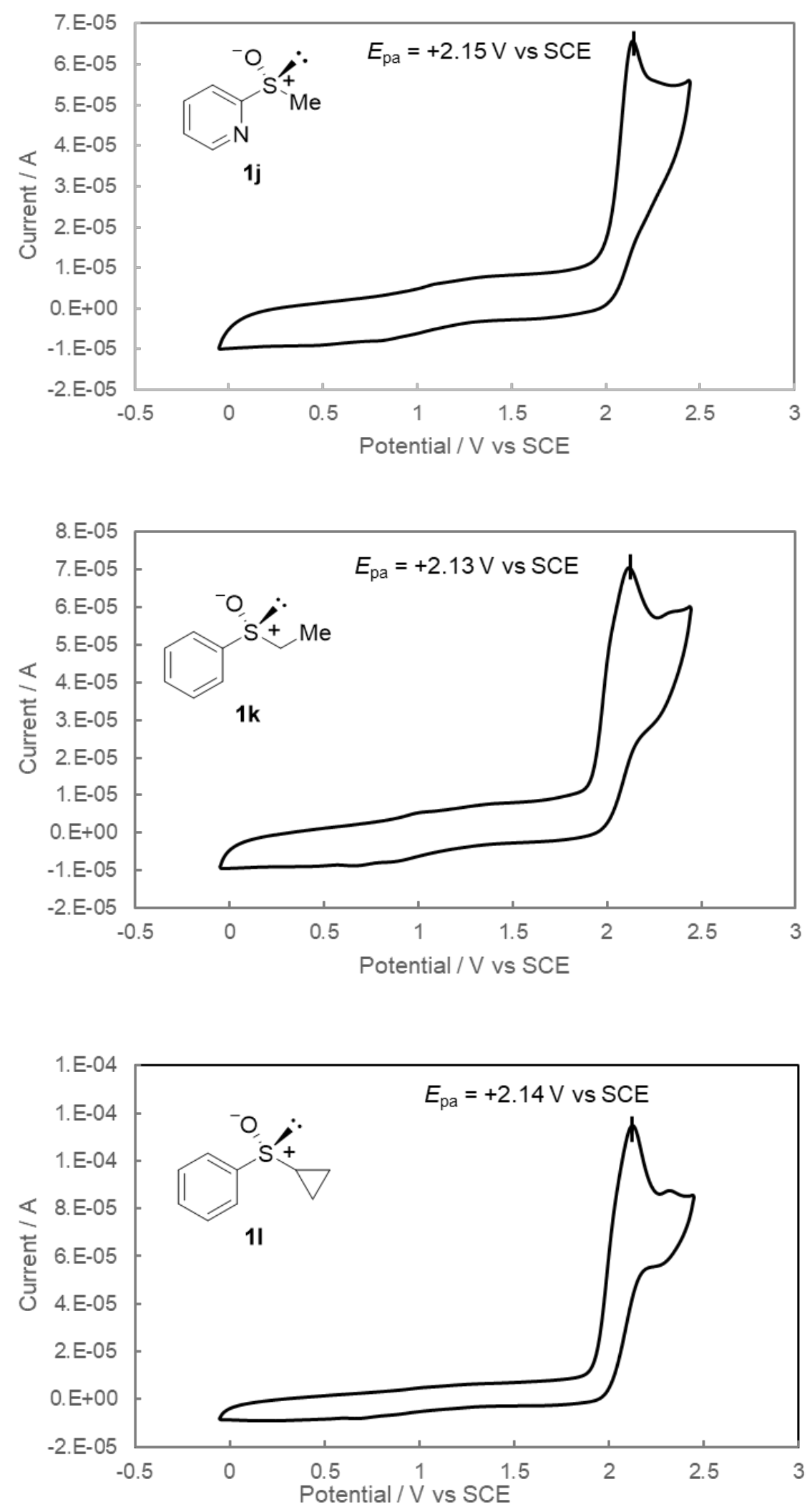

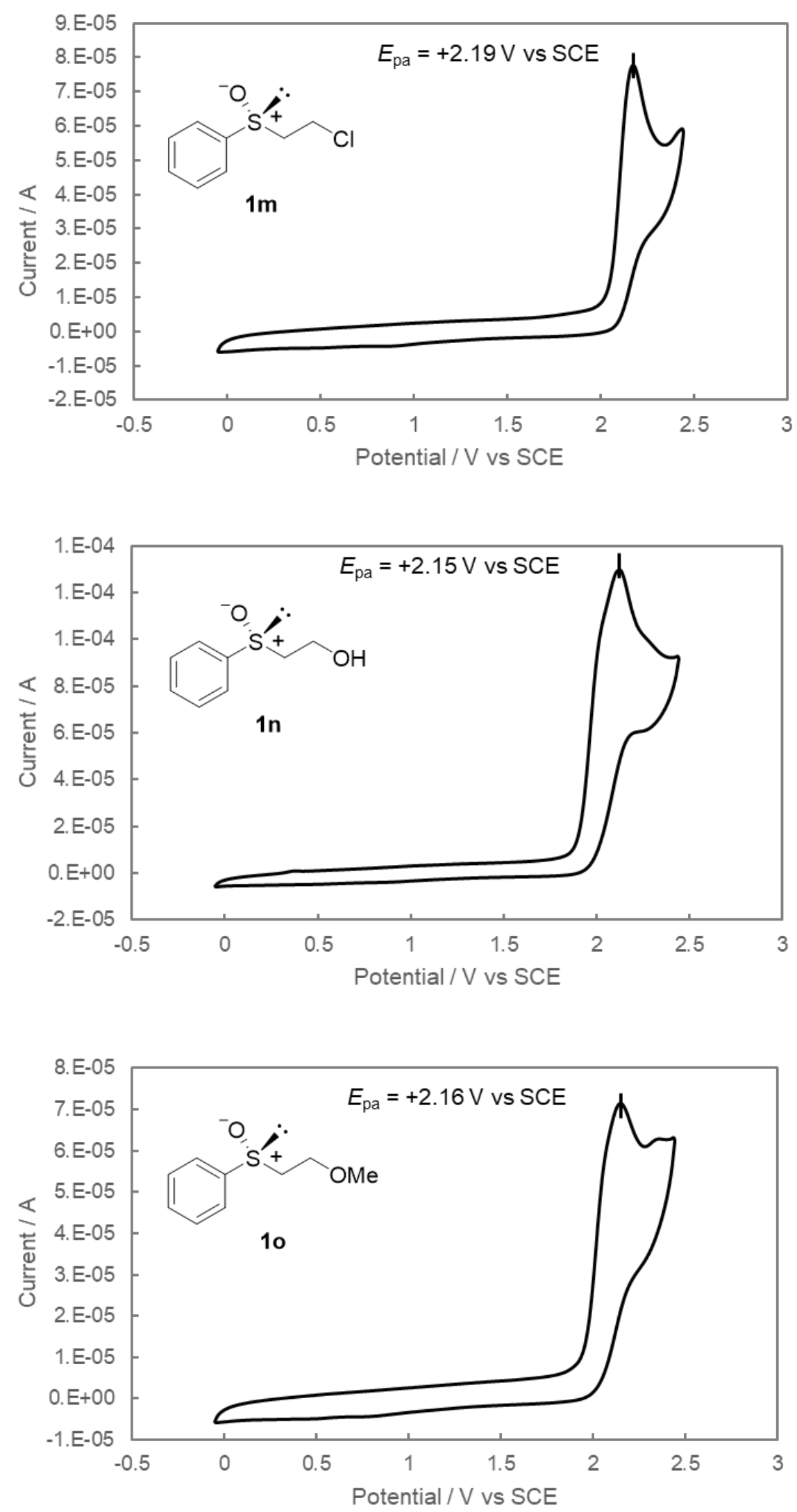

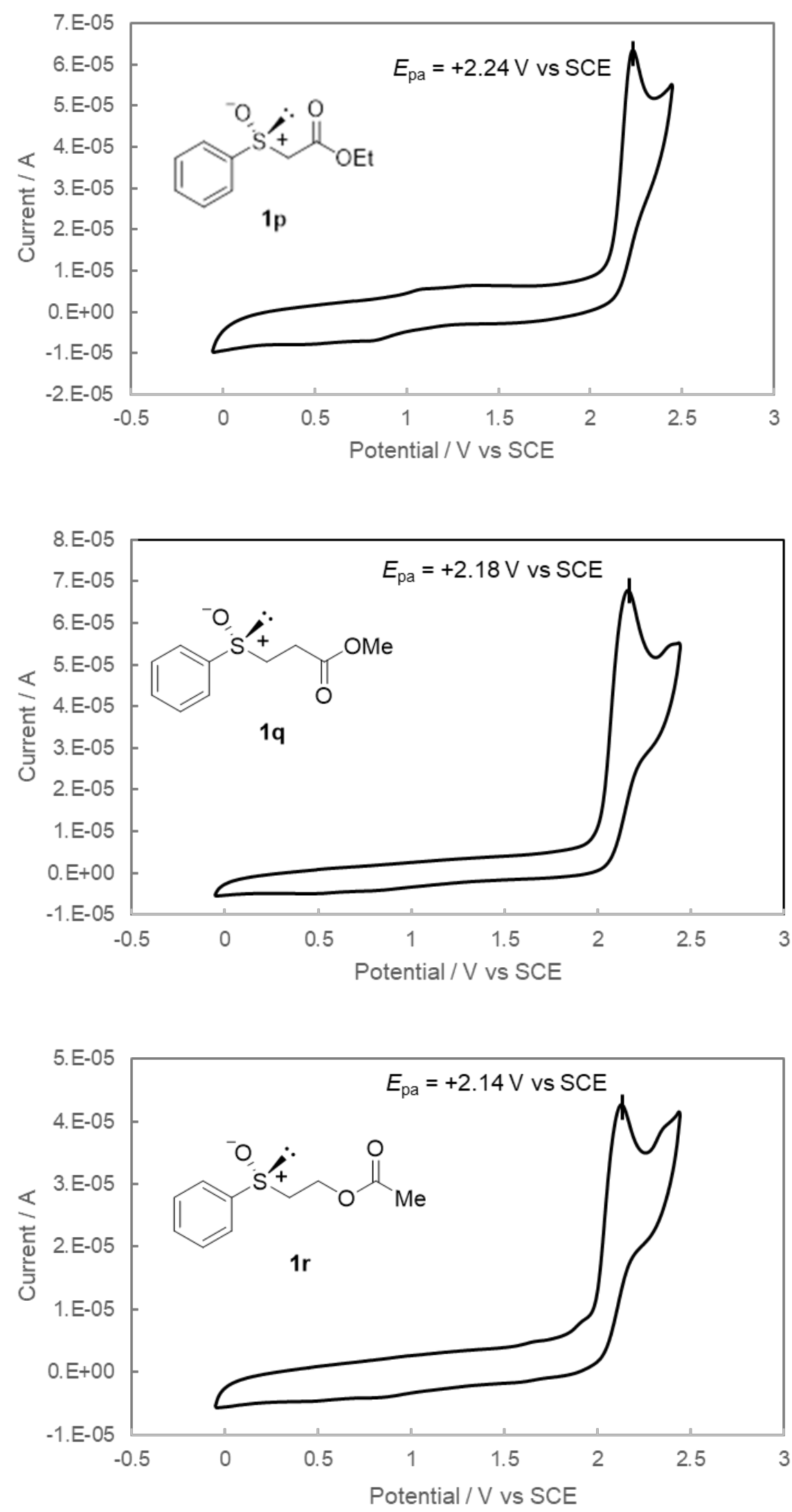

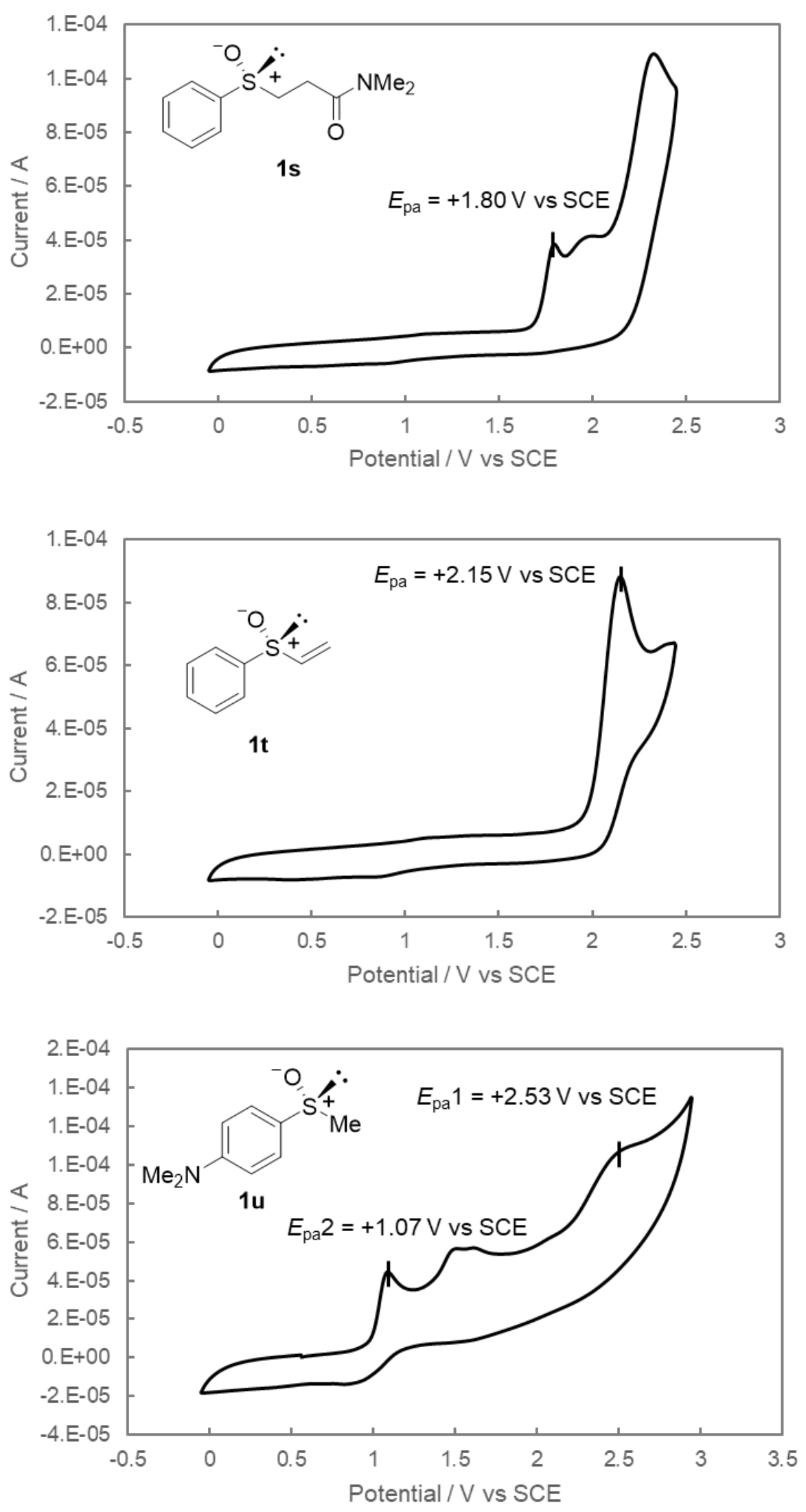

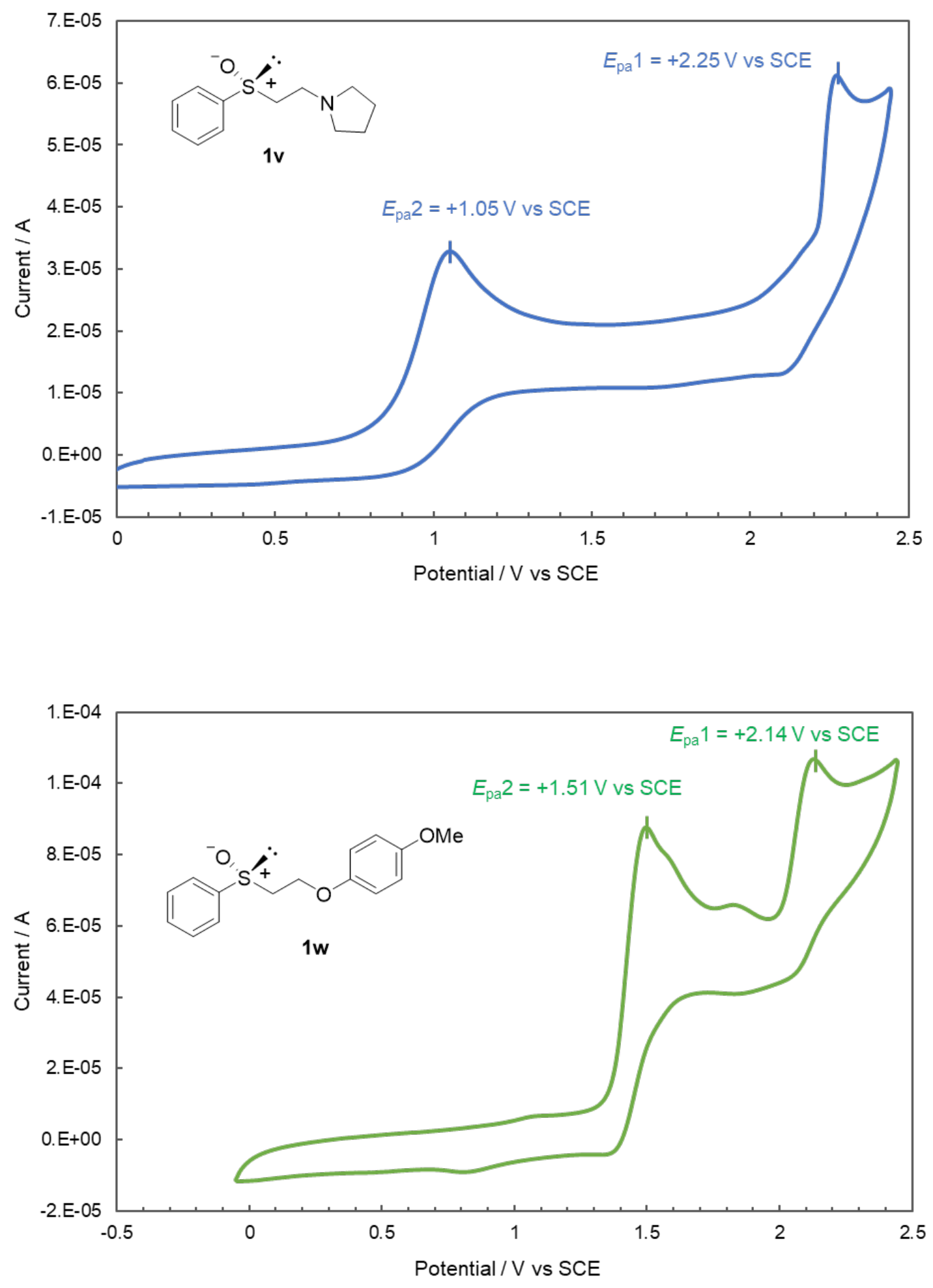

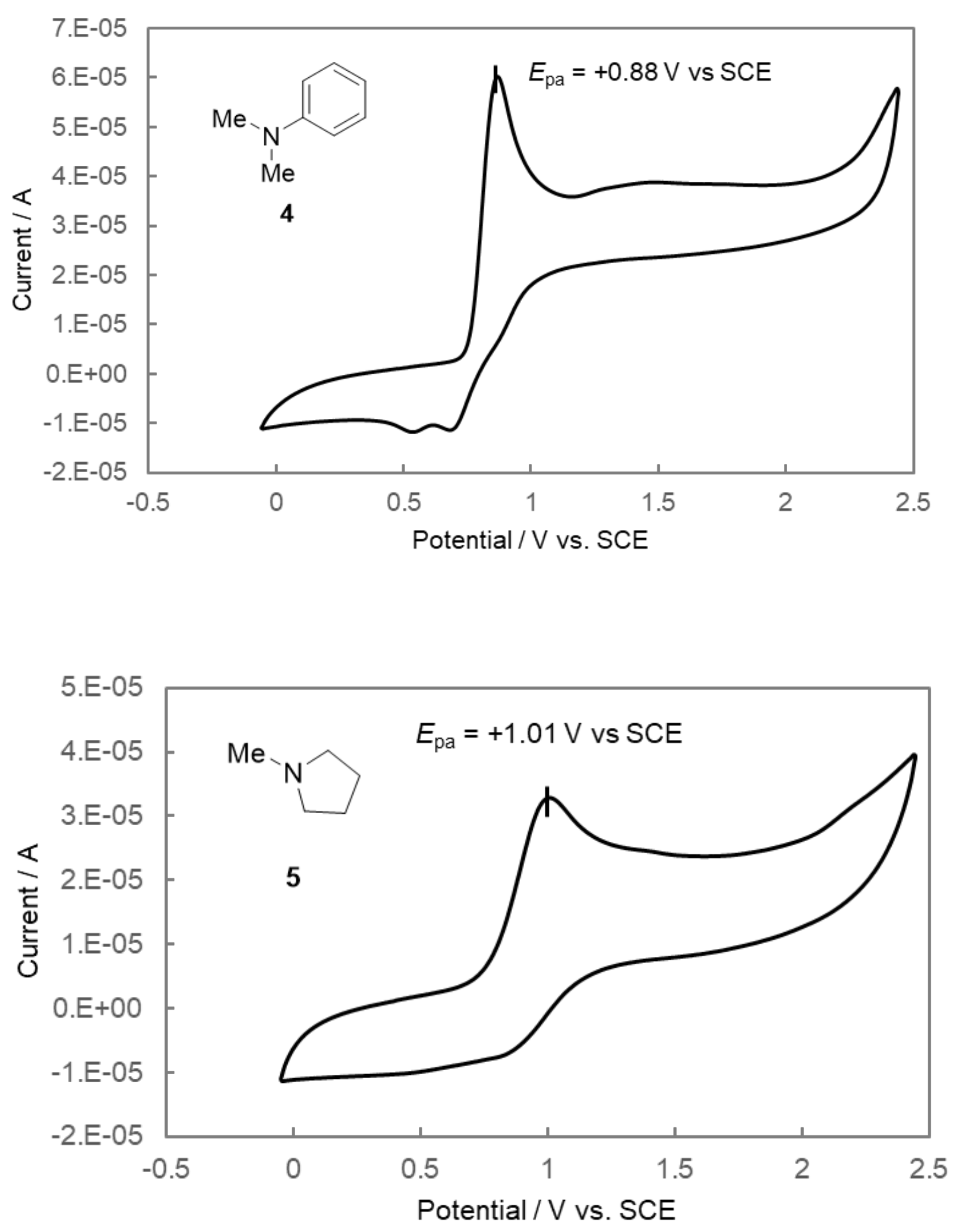


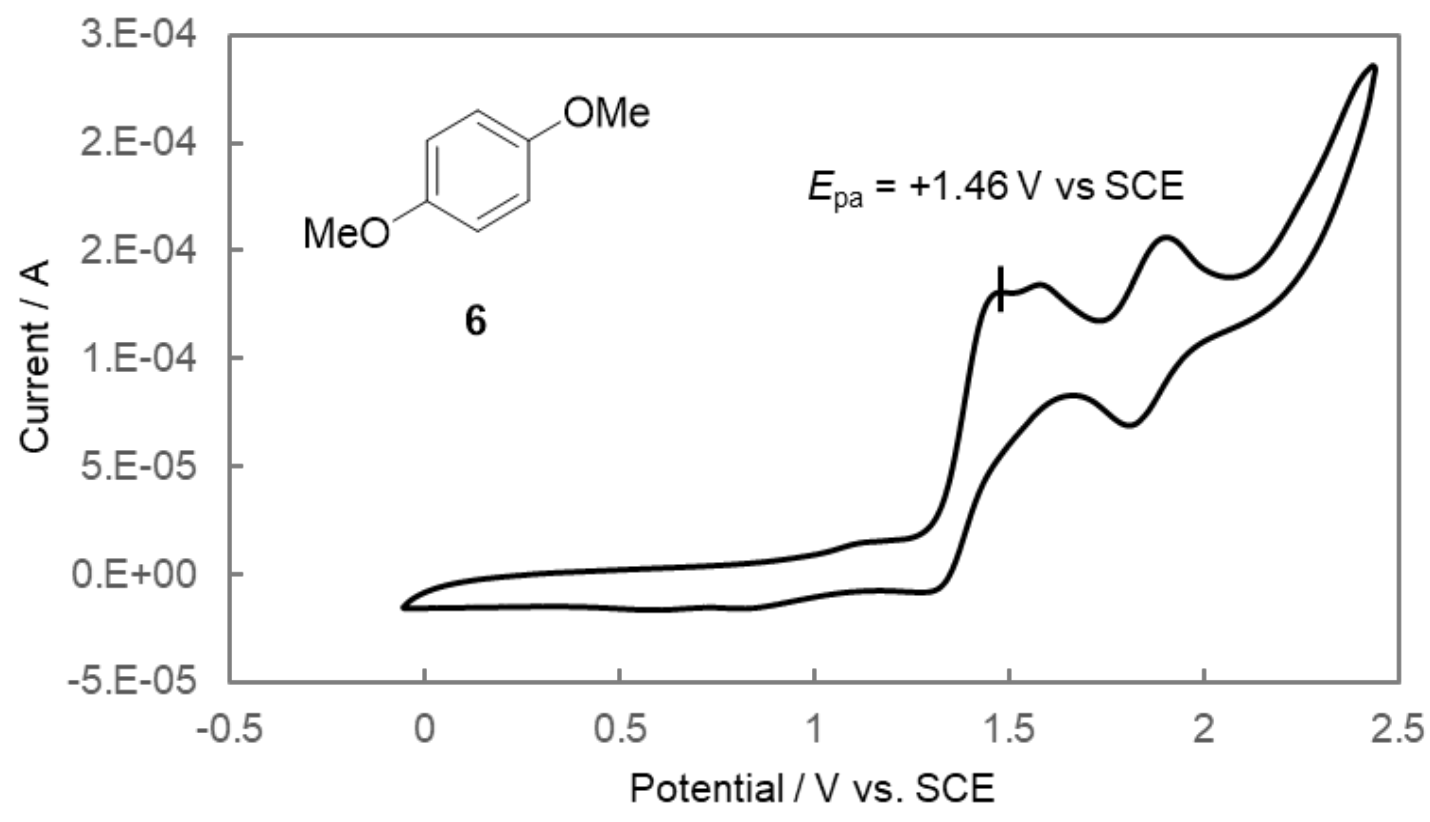




\section{Computational details}

DFT calculations were performed using the Gaussian-16 Revision A.03 quantum chemistry program package using M05-2X functional with the 6-311+G(3df,2p) basis set. The structures of $(+)-1$ a and $(+)-\mathbf{1} \mathbf{a}^{++}$were optimized as a singlet for $(+)-\mathbf{1 a}$ and doublet ground state for $(+)-\mathbf{1} \mathbf{a}^{++}$.

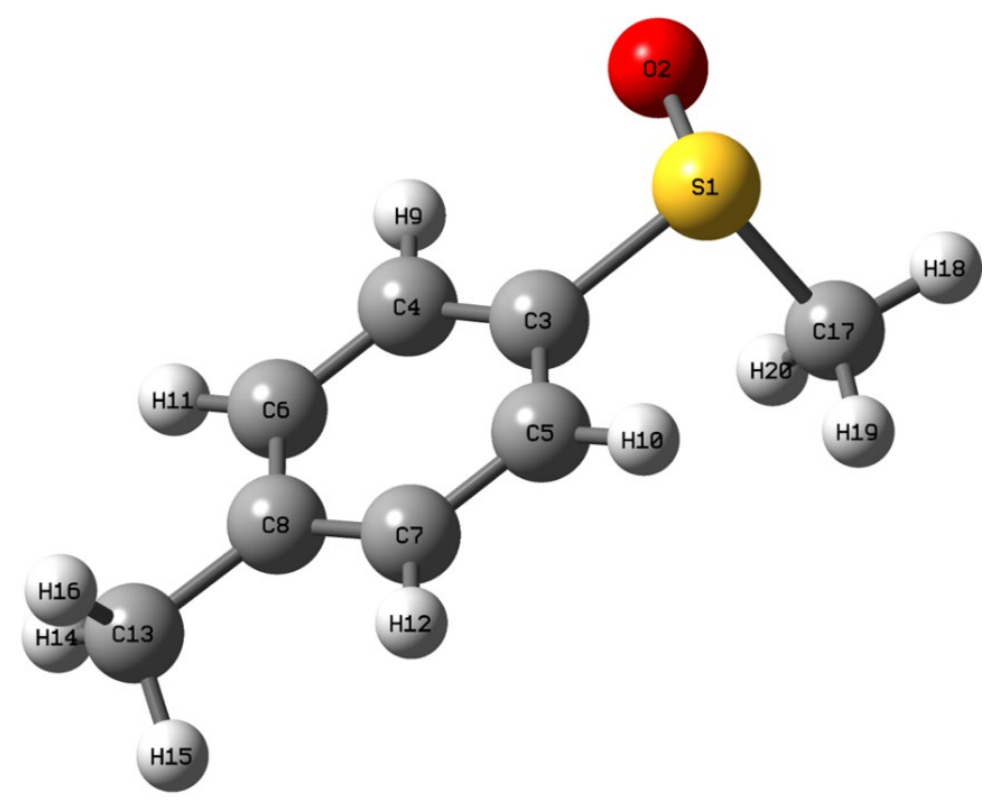

Figure S2. Optimized structure of (+)-1a (neutral).

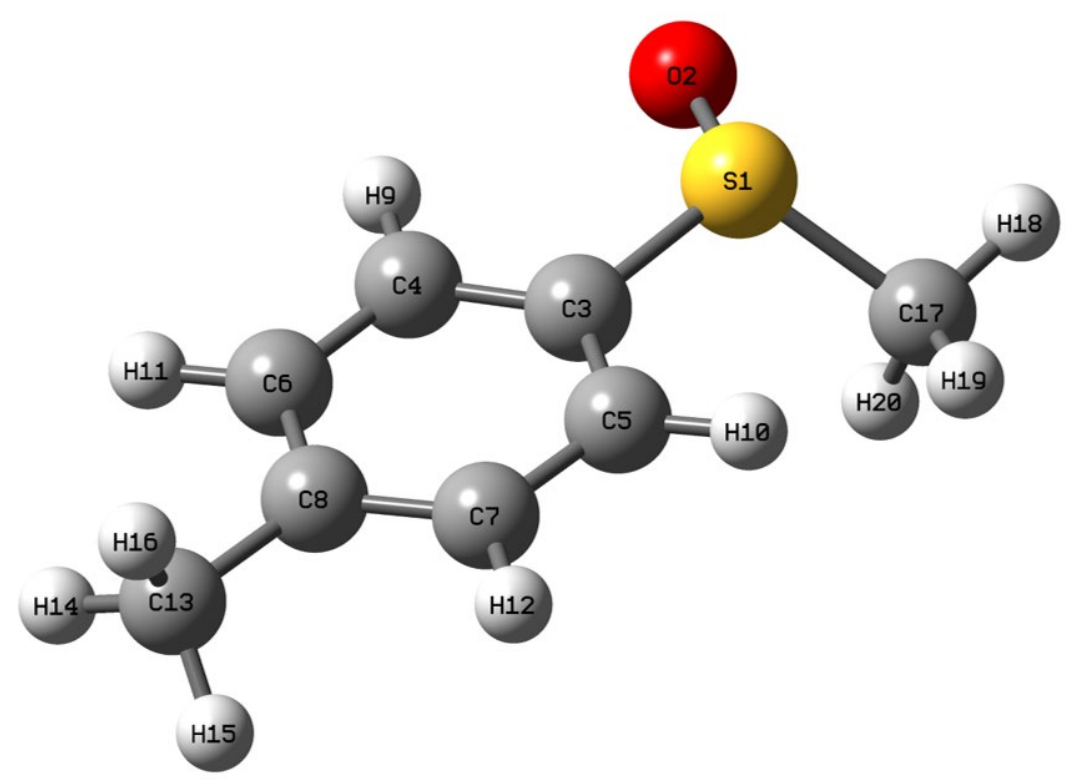

Figure S3. Optimized structure of $(+)-1 \mathbf{a}^{++}$. 
Table S2. XYZ coordinates of the optimized structure of (+)-1a.

\begin{tabular}{|c|c|c|c|}
\hline $\mathrm{E}(\mathrm{RM} 052 \mathrm{X})=$ & 833 & & \\
\hline Item & Value & Threshold & Converged? \\
\hline Maximum Force & 0.000034 & 0.000450 & YES \\
\hline Force & 0.000009 & 0.000300 & YES \\
\hline
\end{tabular}

Predicted change in Energy $=-1.810524 \mathrm{D}-08$

$\begin{array}{lrrr}16 & 2.120779000 & 0.122897000 & -0.430546000 \\ 8 & 2.609655000 & -1.276711000 & -0.304801000 \\ 6 & 0.344023000 & 0.073460000 & -0.235236000 \\ 6 & -0.250176000 & -1.123416000 & 0.111855000 \\ 6 & -0.413875000 & 1.212768000 & -0.456749000 \\ 6 & -1.628895000 & -1.170597000 & 0.259419000 \\ 6 & -1.786404000 & 1.150842000 & -0.299266000 \\ 6 & -2.412212000 & -0.041049000 & 0.059662000 \\ 1 & 0.371712000 & -1.994553000 & 0.252406000 \\ 1 & 0.061652000 & 2.137228000 & -0.753459000 \\ 1 & -2.103515000 & -2.101765000 & 0.532508000 \\ 1 & -2.384819000 & 2.035099000 & -0.464331000 \\ 6 & -3.907672000 & -0.102531000 & 0.193580000 \\ 1 & -4.213005000 & -0.980382000 & 0.755499000 \\ 1 & -4.289824000 & 0.780972000 & 0.698961000 \\ 1 & -4.377237000 & -0.151557000 & -0.787711000 \\ 6 & 2.470887000 & 0.901029000 & 1.156743000 \\ 1 & 3.549242000 & 0.999582000 & 1.227006000 \\ 1 & 1.992197000 & 1.875153000 & 1.193296000 \\ 1 & 2.089846000 & 0.244527000 & 1.932932000\end{array}$

Table S3. XYZ coordinates of the optimized structure of $(+)-\mathbf{1} \mathbf{a}^{\mathbf{*}}$.

$\begin{array}{lrcc}\mathrm{E}(\mathrm{UM} 052 \mathrm{X})= & -784.049198819 & \text { A.U. } & \\ \text { Item } & \text { Value } & \text { Threshold } & \text { Converged? } \\ \text { Maximum Force } & 0.000021 & 0.000450 & \text { YES } \\ \text { RMS Force } & 0.000003 & 0.000300 & \text { YES } \\ \text { Predicted change in Energy=-8.333367D-09 } & \\ \text { 16 } & & \\ & -2.019738000 & 0.063083000 & -0.326972000\end{array}$




$\begin{array}{lrrr}8 & -2.612252000 & 1.363771000 & -0.061211000 \\ 6 & -0.309982000 & 0.014933000 & -0.133739000 \\ 6 & 0.358515000 & 1.220105000 & 0.090551000 \\ 6 & 0.367437000 & -1.197550000 & -0.314833000 \\ 6 & 1.729361000 & 1.185352000 & 0.200411000 \\ 6 & 1.735520000 & -1.197164000 & -0.200708000 \\ 6 & 2.438665000 & -0.012489000 & 0.056259000 \\ 1 & -0.194747000 & 2.139577000 & 0.198552000 \\ 1 & -0.160291000 & -2.111993000 & -0.537948000 \\ 1 & 2.267833000 & 2.097436000 & 0.405828000 \\ 1 & 2.280436000 & -2.121202000 & -0.317277000 \\ 6 & 3.928078000 & -0.032785000 & 0.144214000 \\ 1 & 4.310822000 & 0.858958000 & 0.628610000 \\ 1 & 4.272646000 & -0.911165000 & 0.683383000 \\ 1 & 4.349319000 & -0.080597000 & -0.860722000 \\ 6 & -2.773243000 & -1.221576000 & 0.664155000 \\ 1 & -3.834409000 & -1.172804000 & 0.442295000 \\ 1 & -2.352146000 & -2.176452000 & 0.369912000 \\ 1 & -2.562121000 & -0.982102000 & 1.702194000\end{array}$




\section{Chiral HPLC charts of $1 a-y$ and their optical properties.}

For 1a<smiles>Cc1ccc([S+](C)(C)O)cc1</smiles>

Conditions

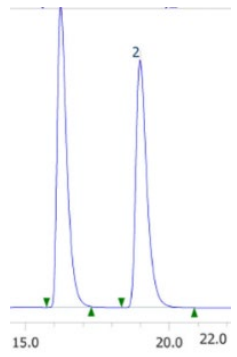

Column: CHIRALPAK IG $(4.6 \mathrm{~mm} \phi \times 250 \mathrm{~mm})$

Eluent: $100 \%$ acetonitrile

Flow rate: $0.5 \mathrm{~mL} / \mathrm{min}$

Detection: $254 \mathrm{~nm}$

Temperature: $23^{\circ} \mathrm{C}$

Former peak $(R): \mathrm{tR}=16.2 \mathrm{~min} ;[\alpha]_{\mathrm{D}}{ }^{20}+184.2$. as $99 \%$ ee, $(c 0.110, \mathrm{MeCN})$

Latter peak $(S)$ : $\mathrm{tR}=19.0 \mathrm{~min} ;[\alpha]_{\mathrm{D}^{20}}-161.9$ as $93 \%$ ee $(c 0.150, \mathrm{MeCN})$

For $\mathbf{1 b}$<smiles>CO[Sb](C)(C)(O)c1ccccc1</smiles>

$1 b$

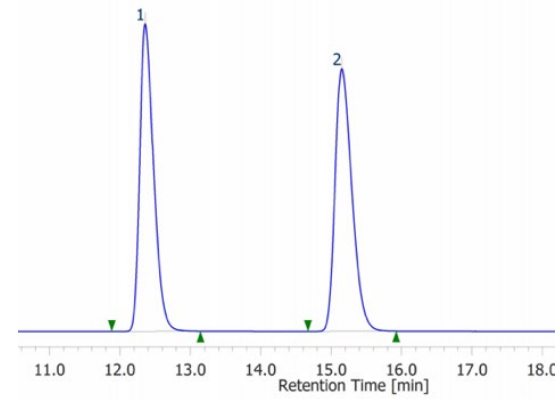

Conditions

Column: CHIRALPAK IH $(4.6 \mathrm{~mm} \phi \times 250 \mathrm{~mm})$

Eluent: $100 \%$ acetonitrile

Flow rate: $0.5 \mathrm{~mL} / \mathrm{min}$

Detection: $254 \mathrm{~nm}$

Temperature: $23^{\circ} \mathrm{C}$

Former peak $(R): \mathrm{tR}=12.4 \mathrm{~min},[\alpha]_{\mathrm{D}}{ }^{20}+134.2$ as $99 \%$ ee $(c 0.200, \mathrm{MeCN})$

Latter peak $(S)$ : $\mathrm{tR}=15.1 \mathrm{~min},[\alpha]_{\mathrm{D}}{ }^{20}-135.3$ as $98 \%$ ee $(c 0.210, \mathrm{MeCN})$ 
For 1c<smiles>COc1ccc([S+](C)(C)C)cc1</smiles>

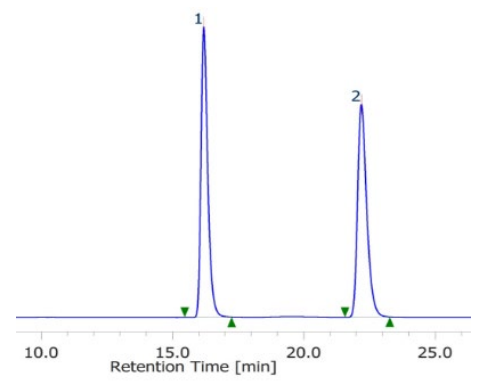

Conditions

Column: CHIRALPAK IH $(4.6 \mathrm{~mm} \phi \times 250 \mathrm{~mm})$

Eluent: $100 \%$ acetonitrile

Flow rate: $0.5 \mathrm{~mL} / \mathrm{min}$

Detection: $254 \mathrm{~nm}$

Temperature: $23{ }^{\circ} \mathrm{C}$

Former peak $(R): \mathrm{tR}=16.6 \mathrm{~min} ;[\alpha]_{\mathrm{D}}{ }^{20}+124.7$ as $99 \%$ ee $(c 0.480, \mathrm{MeCN})$

Latter peak $(S)$ : $\mathrm{tR}=23.1 \mathrm{~min} ;[\alpha]_{\mathrm{D}^{20}}-124.9$ as $97 \%$ ee $(c 0.490, \mathrm{MeCN})$

For 1d<smiles>C[As](C)(C)c1ccc(C=O)cc1</smiles>

1d

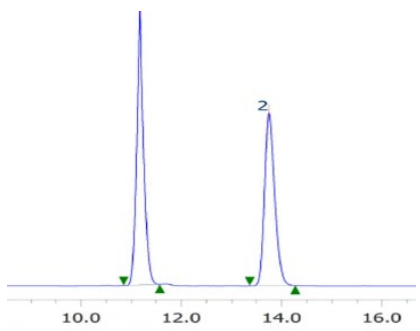

\section{Conditions}

Column: CHIRALPAK IH $(4.6 \mathrm{~mm} \phi \times 250 \mathrm{~mm})$

Eluent: $100 \%$ acetonitrile

Flow rate: $0.5 \mathrm{~mL} / \mathrm{min}$

Detection: $254 \mathrm{~nm}$

Temperature: $23^{\circ} \mathrm{C}$

Former peak $(R)$ : $\mathrm{tR}=11.2 \mathrm{~min} ;[\alpha]_{\mathrm{D}}{ }^{20}+41.1$ as $94 \%$ ee, $(c 0.310, \mathrm{MeCN})$

Latter peak $(S): \mathrm{tR}=13.8 \mathrm{~min} ;[\alpha]_{\mathrm{D}}{ }^{20}-41.6$ as $98 \%$ ee $(c 0.290, \mathrm{MeCN})$ 
For 1e

Conditions

Column: CHIRALPAK IH $(4.6 \mathrm{~mm} \phi \times 250 \mathrm{~mm})$

Eluent: $100 \%$ acetonitrile

Flow rate: $0.5 \mathrm{~mL} / \mathrm{min}$

Detection: $254 \mathrm{~nm}$

Temperature: $23^{\circ} \mathrm{C}$

Former peak $(R)$ : $\mathrm{tR}=12.5 \mathrm{~min} ;[\alpha]_{\mathrm{D}}{ }^{20}-107.4$ as $99 \%$ ee, $(c 0.260, \mathrm{MeCN})$

Latter peak $(S): \mathrm{tR}=14.6 \mathrm{~min} ;[\alpha]_{\mathrm{D}^{20}}+106.7$ as $96 \%$ ee $(c 0.350, \mathrm{MeCN})$

For $\mathbf{1 f}$<smiles>C[S+](C)(O)c1ccc(Cl)cc1</smiles>

Conditions

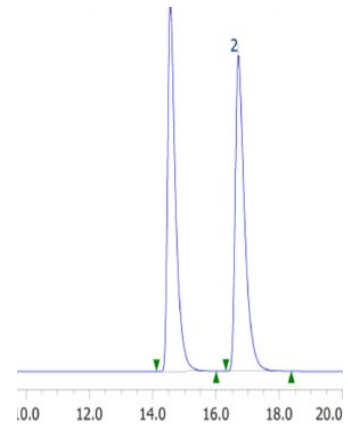

Column: CHIRALPAK IH $(4.6 \mathrm{~mm} \phi \times 250 \mathrm{~mm})$

Eluent: $100 \%$ acetonitrile

Flow rate: $0.5 \mathrm{~mL} / \mathrm{min}$

Detection: $254 \mathrm{~nm}$

Temperature: $23^{\circ} \mathrm{C}$

Former peak $(R): \mathrm{tR}=14.6 \mathrm{~min} ;[\alpha]_{\mathrm{D}}{ }^{20}+98.2$ as $99 \%$ ee, $(c 0.320, \mathrm{MeCN})$

Latter peak $(S)$ : $\mathrm{tR}=16.7 \mathrm{~min} ;[\alpha]_{\mathrm{D}}{ }^{20}-95.7$ as $99 \%$ ee $(c 0.300, \mathrm{MeCN})$ 
For 19<smiles>C[As](C)(C)c1ccc(F)cc1</smiles>

$1 \mathrm{~g}$

$\underline{\text { Conditions }}$

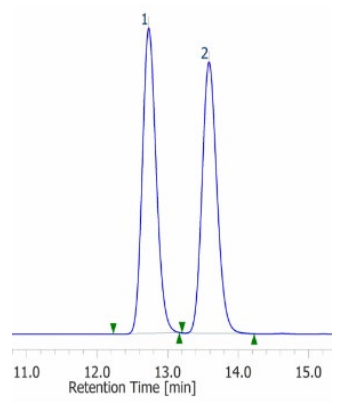

Column: CHIRALPAK IH $(4.6 \mathrm{~mm} \Phi \times 250 \mathrm{~mm})$

Eluent: $100 \%$ acetonitrile

Flow rate: $0.5 \mathrm{~mL} / \mathrm{min}$

Detection: $254 \mathrm{~nm}$

Temperature: $23{ }^{\circ} \mathrm{C}$

Former peak $(R)$ : $\mathrm{tR}=12.7 \mathrm{~min} ;[\alpha]_{\mathrm{D}}{ }^{20}+121.7$ as $99 \%$ ee, $(c 0.080, \mathrm{MeCN})$

Latter peak $(S)$ : $\mathrm{tR}=13.6 \mathrm{~min} ;[\alpha]_{\mathrm{D}}^{20}-105.2$ as $96 \%$ ee $(c 0.165, \mathrm{MeCN})$

\section{For $1 \mathrm{~h}$}<smiles>CO[S-](C)(C)c1cccc(F)c1</smiles>

$1 \mathrm{~h}$

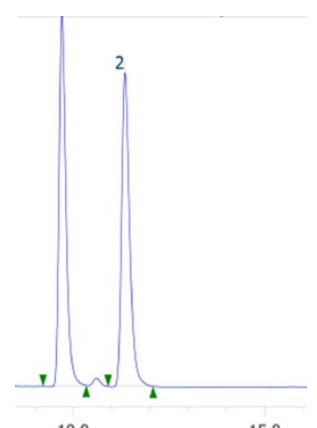

Conditions

10.0

Column: CHIRALPAK IG $(4.6 \mathrm{~mm} \phi \times 250 \mathrm{~mm})$

Eluent: $100 \%$ acetonitrile

Flow rate: $0.5 \mathrm{~mL} / \mathrm{min}$

Detection: $254 \mathrm{~nm}$

Temperature: $23^{\circ} \mathrm{C}$

Former peak $(R): \mathrm{tR}=9.7 \mathrm{~min} ;[\alpha]_{\mathrm{D}}{ }^{20}+110.8$ as $99 \%$ ee, $(c 0.240, \mathrm{MeCN})$

Latter peak $(S)$ : $\mathrm{tR}=11.4 \mathrm{~min} ;[\alpha]_{\mathrm{D}}^{20}-111.7$ as $98 \%$ ee $(c 0.200, \mathrm{MeCN})$ 


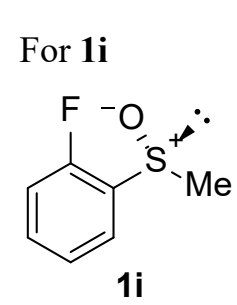

\section{Conditions}

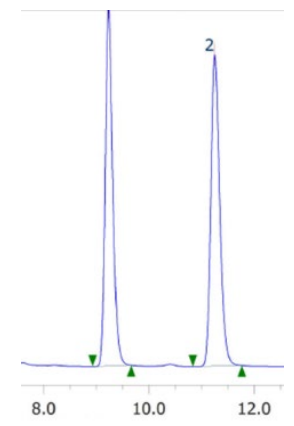

Column: CHIRALPAK IH $(4.6 \mathrm{~mm} \phi \times 250 \mathrm{~mm})$

Eluent: $100 \%$ acetonitrile

Flow rate: $0.5 \mathrm{~mL} / \mathrm{min}$

Detection: $254 \mathrm{~nm}$

Temperature: $23^{\circ} \mathrm{C}$

Former peak $(R)$ : $\mathrm{tR}=9.2 \mathrm{~min} ;[\alpha]_{\mathrm{D}}{ }^{20}+162.2$ as $99 \%$ ee $(c 0.210, \mathrm{MeCN})$

Latter peak $(S)$ : $\mathrm{tR}=11.2 \mathrm{~min} ;[\alpha]_{\mathrm{D}}{ }^{20}-162.5$ as $98 \%$ ee $(c 0.130, \mathrm{MeCN})$<smiles>C[S+](C)(O)c1ccccn1</smiles>

Conditions

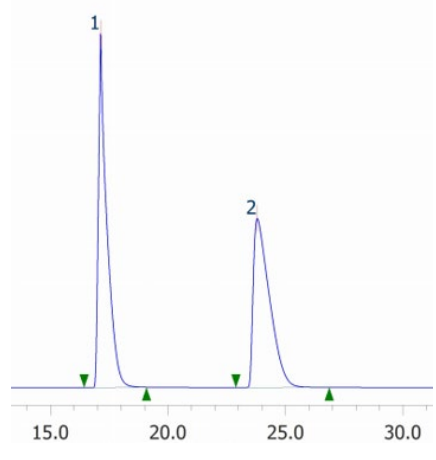

Column: CHIRALPAK IH $(4.6 \mathrm{~mm} \phi \times 250 \mathrm{~mm})$

Eluent: $40 \% i$-PrOH in hexane,

Flow rate: $0.5 \mathrm{~mL} / \mathrm{min}$

Detection: $254 \mathrm{~nm}$

Temperature: $23^{\circ} \mathrm{C}$

Former peak $(R)$ : $\mathrm{tR}=17.1 \mathrm{~min} ;[\alpha]_{\mathrm{D}}{ }^{20}+59.3$ as $99 \%$ ee $(c 0.110, \mathrm{MeCN})$

Latter peak $(S)$ : $\mathrm{tR}=23.8 \mathrm{~min} ;[\alpha]_{\mathrm{D}}{ }^{20}-58.9$ as $99 \%$ ee $(c 1.320, \mathrm{MeCN})$ 
For 1k<smiles>CC[S+](C)(O)c1ccccc1</smiles>

Conditions

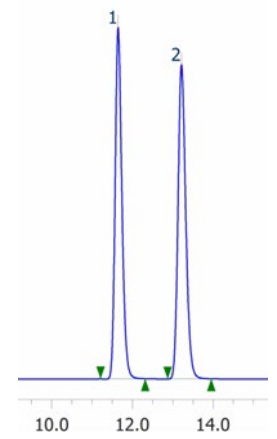

Column: CHIRALPAK IH $(4.6 \mathrm{~mm} \phi \times 250 \mathrm{~mm})$

Eluent: $100 \%$ acetonitrile

Flow rate: $0.5 \mathrm{~mL} / \mathrm{min}$

Detection: $254 \mathrm{~nm}$

Temperature: $23{ }^{\circ} \mathrm{C}$,

Former peak $(R): \mathrm{tR}=11.6 \min [\alpha]_{\mathrm{D}}{ }^{20}+142.1$ as $99 \%$ ee $(c 0.150, \mathrm{MeCN})$

Latter peak $(S)$ : $\mathrm{tR}=13.2 \mathrm{~min}[\alpha]_{\mathrm{D}}{ }^{20}-142.9$ as $96 \%$ ee $(c 0.220, \mathrm{MeCN})$<smiles>[3H][S+]([O-])(c1ccccc1)C1CC1</smiles>

Conditions

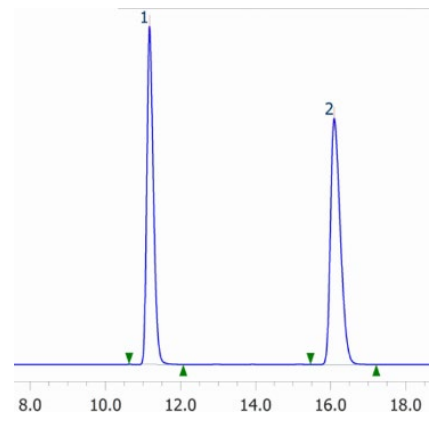

Column: CHIRALPAK IH $(4.6 \mathrm{~mm} \phi \times 250 \mathrm{~mm})$

Eluent: $100 \%$ acetonitrile

Flow rate: $0.5 \mathrm{~mL} / \mathrm{min}$

Detection: $254 \mathrm{~nm}$

Temperature: $23^{\circ} \mathrm{C}$

Former peak $(R)$ : $\mathrm{tR}=11.2 \mathrm{~min} ;[\alpha]_{\mathrm{D}}^{20}+194.7$ as $97 \%$ ee $(c 0.160, \mathrm{MeCN})$

Latter peak $(S)$ : $\mathrm{tR}=16.1 \mathrm{~min} ;[\alpha]_{\mathrm{D}}^{20}-195.6$ as $99 \%$ ee $(c 0.160, \mathrm{MeCN})$ 


\section{For $1 \mathrm{~m}$}<smiles>C[Sb](O)(CCCl)c1ccccc1</smiles>

$1 \mathrm{~m}$

\section{Conditions}

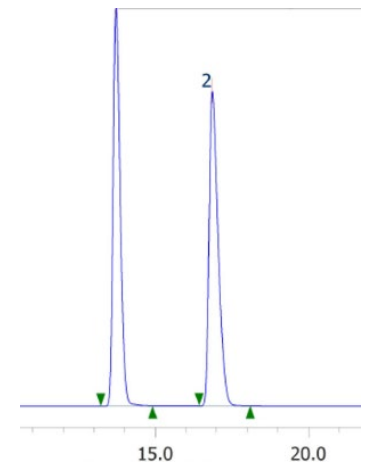

Column: CHIRALPAK IG $(4.6 \mathrm{~mm} \phi \times 250 \mathrm{~mm})$

Eluent: $100 \%$ acetonitrile

Flow rate: $0.5 \mathrm{~mL} / \mathrm{min}$

Detection: $254 \mathrm{~nm}$

Temperature: $23{ }^{\circ} \mathrm{C}$

Former peak $(R): \mathrm{tR}=14.8 \mathrm{~min} ;[\alpha]_{\mathrm{D}}{ }^{20}+102.7$ as $99 \%$ ee $(c 0.310, \mathrm{MeCN})$

Latter peak $(S): \mathrm{tR}=18.3 \mathrm{~min} ;[\alpha]_{\mathrm{D}^{20}}-104.5$ as $99 \%$ ee $(c 0.520, \mathrm{MeCN})$<smiles>C[Si](CCO)(CCO)c1ccccc1</smiles>

$\underline{\text { Conditions }}$

\section{Column: CHIRALPAK IF $(4.6 \mathrm{~mm} \phi \times 250 \mathrm{~mm})$}

Eluent: $100 \%$ acetonitrile

Flow rate: $0.5 \mathrm{~mL} / \mathrm{min}$

Detection: $254 \mathrm{~nm}$

Temperature: $23{ }^{\circ} \mathrm{C}$

Former peak $(R): \mathrm{tR}=13.4 \mathrm{~min} ;[\alpha]_{\mathrm{D}}{ }^{20}+225.6$ as $98 \%$ ee $(c 0.410, \mathrm{MeCN})$

Latter peak $(S)$ : $\mathrm{tR}=18.0 \mathrm{~min} ;[\alpha]_{\mathrm{D}}^{20}-227.1$ as $97 \%$ ee $(c 0.310, \mathrm{MeCN})$ 

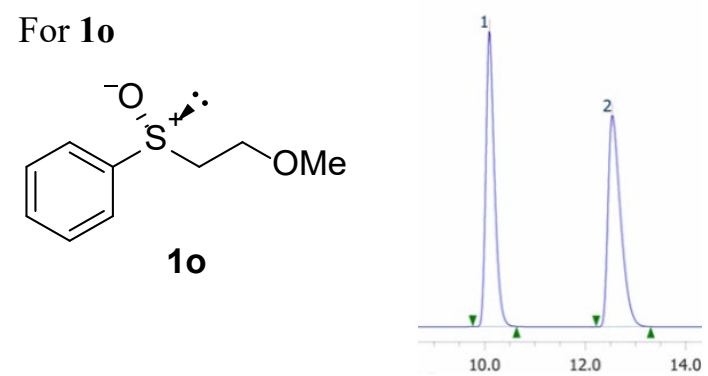

Conditions

Column: CHIRALPAK ID $(4.6 \mathrm{~mm} \phi \times 250 \mathrm{~mm})$

Eluent: $100 \%$ acetonitrile

Flow rate: $0.5 \mathrm{~mL} / \mathrm{min}$

Detection: $254 \mathrm{~nm}$

Temperature: $23{ }^{\circ} \mathrm{C}$

Former peak $(R): \mathrm{tR}=10.1 \mathrm{~min} ;[\alpha]_{\mathrm{D}}{ }^{20}+209.0$ as $99 \%$ ee $(c 0.130, \mathrm{MeCN})$

Latter peak $(S)$ : $\mathrm{tR}=12.5 \mathrm{~min} ;[\alpha]_{\mathrm{D}^{20}}-207.2$ as $99 \%$ ee $(c 0.150, \mathrm{MeCN})$<smiles>CCOC(=O)C[S+](C)(C)c1ccccc1</smiles>

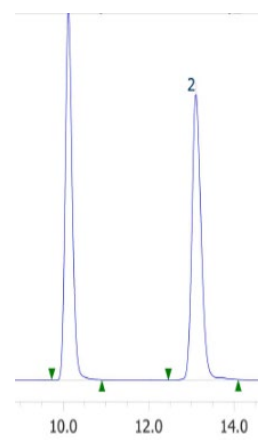

\section{Conditions}

Column: CHIRALPAK IG $(4.6 \mathrm{~mm} \phi \times 250 \mathrm{~mm})$

Eluent: $100 \%$ acetonitrile

Flow rate: $0.5 \mathrm{~mL} / \mathrm{min}$

Detection: $254 \mathrm{~nm}$

Temperature: $23{ }^{\circ} \mathrm{C}$

Former peak $(R)$ : $\mathrm{tR}=10.1 \mathrm{~min} ;[\alpha]_{\mathrm{D}}{ }^{20}-204.4$ as $99 \%$ ee $(c 0.360, \mathrm{MeCN})$.

Latter peak $(S)$ : $\mathrm{tR}=13.1 \mathrm{~min} ;[\alpha]_{\mathrm{D}}^{20}+183.8$ as $99 \%$ ee $(c 0.290, \mathrm{MeCN})$ 
For 1q<smiles>COC(=O)CC[S+](C)(=O)c1ccccc1</smiles>

$1 q$

Conditions

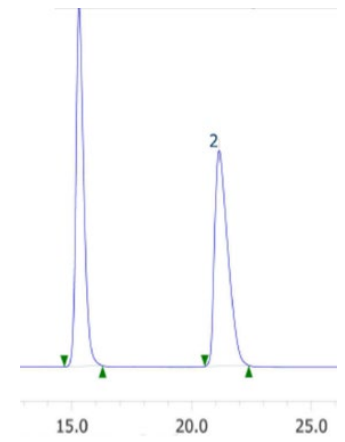

Column: CHIRALPAK IF $(4.6 \mathrm{~mm} \phi \times 250 \mathrm{~mm})$

Eluent: $100 \%$ acetonitrile

Flow rate: $0.5 \mathrm{~mL} / \mathrm{min}$

Detection: $254 \mathrm{~nm}$

Temperature: $23{ }^{\circ} \mathrm{C}$

Former peak $(R)$ : $\mathrm{tR}=15.3 \mathrm{~min} ;[\alpha]_{\mathrm{D}}{ }^{20}+121.1$ as $99 \%$ ee $(c 0.410, \mathrm{MeCN})$

Latter peak $(S)$ : $\mathrm{tR}=21.1 \mathrm{~min} ;[\alpha]_{\mathrm{D}^{20}}-124.6$ as $94 \%$ ee $(c 0.230, \mathrm{MeCN})$<smiles>CC(=O)OCC[Si](C)(C)c1ccccc1</smiles>

Conditions

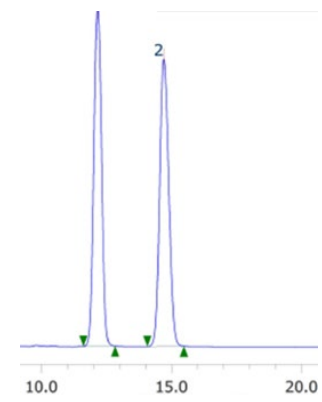

Column: CHIRALPAK IF $(4.6 \mathrm{~mm} \phi \times 250 \mathrm{~mm})$

Eluent: $100 \%$ acetonitrile,

Flow rate: $0.5 \mathrm{~mL} / \mathrm{min}$

Detection: $254 \mathrm{~nm}$

Temperature: $23{ }^{\circ} \mathrm{C}$

Former peak $(R): \mathrm{tR}=12.1 \mathrm{~min} ;[\alpha]_{\mathrm{D}}{ }^{20}-123.3$ as $99 \%$ ee $(c 0.110, \mathrm{MeCN})$

Latter peak $(S)$ : $\mathrm{tR}=14.7 \mathrm{~min} ;[\alpha]_{\mathrm{D}}^{20}+121.7$ as $98 \%$ ee $(c 0.170, \mathrm{MeCN})$ 
For 1s<smiles>Cc1ccc([S+](C)(O)c2ccccc2)cc1</smiles>

$1 \mathrm{~s}$

Conditions

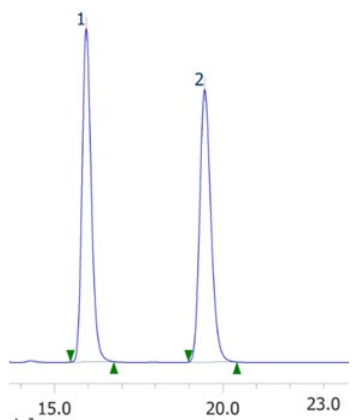

Column: CHIRALPAK IG $(4.6 \mathrm{~mm} \phi \times 250 \mathrm{~mm})$

Eluent: $100 \%$ acetonitrile

Flow rate: $0.5 \mathrm{~mL} / \mathrm{min}$

Detection: $254 \mathrm{~nm}$

Temperature: $23^{\circ} \mathrm{C}$

Former peak $(S)$ : $\mathrm{tR}=15.9 \mathrm{~min} ;[\alpha]_{\mathrm{D}}{ }^{20}-13.8$ as $99 \%$ ee $(c 0.250, \mathrm{MeCN})$

Latter peak $(R)$ : $\mathrm{tR}=19.5 \mathrm{~min} ;[\alpha]_{\mathrm{D}} \mathrm{D}^{20}+18.8$ as $99 \%$ ee $(c 0.250, \mathrm{MeCN})$

For 1t<smiles>C=C[Si](C)(O)c1ccccc1</smiles>

$1 \mathrm{t}$

Conditions

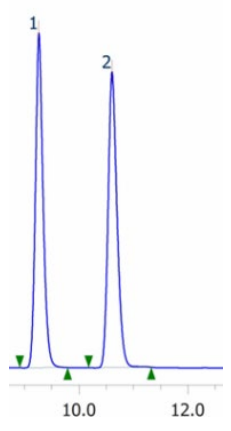

Column: CHIRALPAK IH $(4.6 \mathrm{~mm} \phi \times 250 \mathrm{~mm})$

Eluent: $100 \%$ acetonitrile

Flow rate: $0.5 \mathrm{~mL} / \mathrm{min}$

Detection: $254 \mathrm{~nm}$

Temperature: $23{ }^{\circ} \mathrm{C}$

Former peak $(R): \mathrm{tR}=9.3 \mathrm{~min} ;[\alpha]_{\mathrm{D}}{ }^{20}+421.0$ as $99 \%$ ee $(c 0.310, \mathrm{MeCN})$

Latter peak $(S)$ : $\mathrm{tR}=10.6 \mathrm{~min} ;[\alpha]_{\mathrm{D}}^{20}-418.5$ as $99 \%$ ee $(c 0.190, \mathrm{MeCN})$ 
For 1u

Conditions

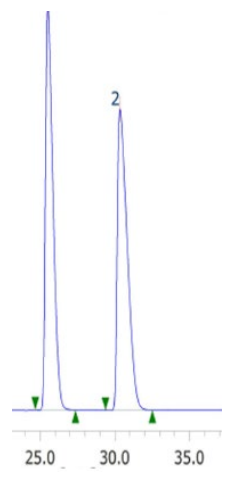

Column: CHIRALPAK IA $(4.6 \mathrm{~mm} \phi \times 250 \mathrm{~mm})$

Eluent: $100 \%$ acetonitrile

Flow rate: $0.5 \mathrm{~mL} / \mathrm{min}$

Detection: $254 \mathrm{~nm}$

Temperature: $23^{\circ} \mathrm{C}$

Former peak $(R)$ : $\mathrm{tR}=25.5 \mathrm{~min} ;[\alpha]_{\mathrm{D}}{ }^{20}-91.6$ as $99 \%$ ee $(c 0.130, \mathrm{MeCN})$

Latter peak $(S): \mathrm{tR}=30.3 \mathrm{~min} ;[\alpha]_{\mathrm{D}^{20}}-102.9$ as $99 \%$ ee $(c 0.060, \mathrm{MeCN})$

For 1v<smiles>C[Sn](C)(CCN1CCCC1)c1ccccc1</smiles>

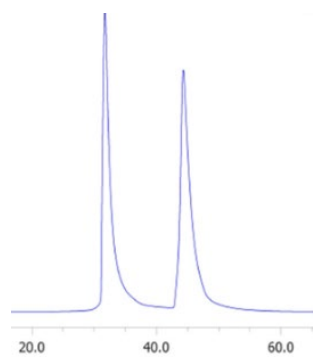

\section{Conditions}

Column: CHIRALPAK IG $(4.6 \mathrm{~mm} \phi \times 250 \mathrm{~mm})$

Eluent: $100 \%$ acetonitrile

Flow rate: $0.5 \mathrm{~mL} / \mathrm{min}$

Detection: $254 \mathrm{~nm}$

Temperature: $23^{\circ} \mathrm{C}$

Former peak: $\mathrm{tR}=31.7 \mathrm{~min} ;[\alpha]_{\mathrm{D}}{ }^{20}+133.8$ as $99 \%$ ee $(c 0.090, \mathrm{MeCN})$.

Latter peak: $\mathrm{tR}=43.3 \mathrm{~min} ;[\alpha]_{\mathrm{D}}{ }^{20}-128.8$ as $99 \%$ ee $(c 0.030, \mathrm{MeCN})$ 
<smiles>COc1ccc(OCC[Si](C)([O-])c2ccccc2)cc1</smiles>

$\underline{\text { Conditions }}$

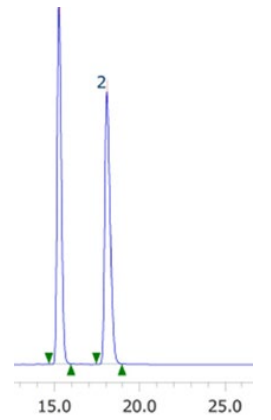

Column: CHIRALPAK IA $(4.6 \mathrm{~mm} \phi \times 250 \mathrm{~mm})$

Eluent: $100 \%$ acetonitrile

Flow rate: $0.5 \mathrm{~mL} / \mathrm{min}$

Detection: $254 \mathrm{~nm}$

Temperature: $23^{\circ} \mathrm{C}$

Former peak: $\mathrm{tR}=15,2 \mathrm{~min} ;[\alpha]_{\mathrm{D}}{ }^{20}+91.5$ as $99 \%$ ee $(c 0.180, \mathrm{MeCN})$.

Latter peak: $\mathrm{tR}=17.9 \mathrm{~min} ;[\alpha]_{\mathrm{D}^{20}}-91.0$ as $95 \%$ ee $(c 0.250, \mathrm{MeCN})$ 


\section{Optical purity of (+)-1a-w.}

For 1a<smiles>Cc1ccc([Si](C)(C)O)cc1</smiles>

$(+)-1 \mathrm{a}$

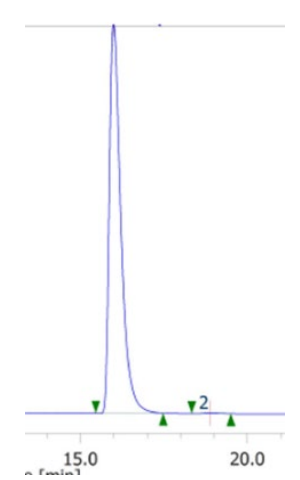

HPLC (CHIRALPAK IG, acetonitrile, flow rate $=0.5$ $\mathrm{mL} / \mathrm{min}, 1=254 \mathrm{~nm}$ ) $\mathrm{tR}=16.0 \mathrm{~min}, 99.8 \%$ (major), 18.9 min, $0.2 \%$ (minor), $99.6 \%$ ee

HPLC $($ CHIRALPAK IH, acetonitrile, flow rate $=$ $0.5 \mathrm{~mL} / \mathrm{min}, 1=254 \mathrm{~nm}) \mathrm{tR}=12.1 \mathrm{~min}, 99.3 \%$ (major), $15.1 \mathrm{~min}, 0.7 \%$ (minor), $98.6 \% \mathrm{ee}$

For 1c<smiles>COc1ccc([S+](C)(C)C)cc1</smiles>

$(+)-1 c$

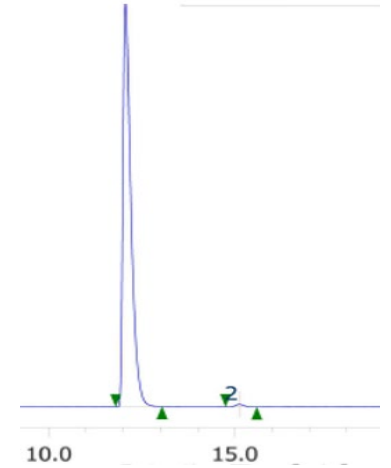

10.0
15.0

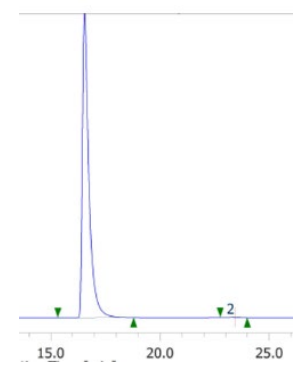

HPLC (CHIRALPAK IH, acetonitrile, flow rate $=$ $0.5 \mathrm{~mL} / \mathrm{min}, 1=254 \mathrm{~nm}) \mathrm{tR}=16.5 \mathrm{~min}, 99.8 \%$ (major), $23.4 \mathrm{~min}, 0.2 \%$ (minor), $99.6 \% \mathrm{ee}$

For 1d<smiles>C[Si](C)(C)c1ccc(C=O)cc1</smiles>

$(+)-1 d$

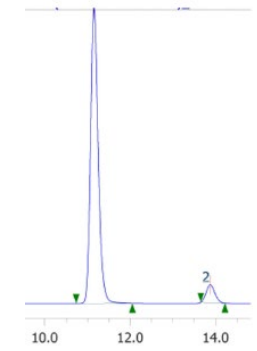

HPLC (CHIRALPAK IH, acetonitrile, flow rate $=0.5$ $\mathrm{mL} / \mathrm{min}, 1=254 \mathrm{~nm}$ ) $\mathrm{tR}=11.2 \mathrm{~min}, 93.5 \%$ (major), $13.9 \min , 6.5 \%$ (minor), $87.0 \%$ ee 
For 1e<smiles>C[S+](C)(O)c1ccc([N+](=O)[O-])cc1</smiles>

$(+)-1 e$

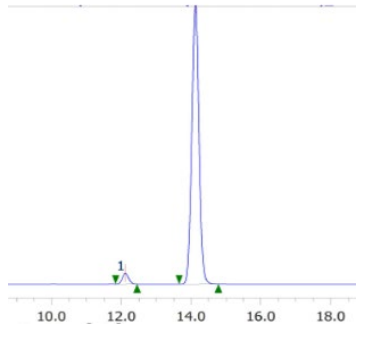

HPLC (CHIRALPAK IH, acetonitrile, flow rate = $0.5 \mathrm{~mL} / \mathrm{min}, 1=254 \mathrm{~nm}) \mathrm{tR}=12.1 \mathrm{~min}, 3.2 \%$ (minor), $14.1 \mathrm{~min}, 96.8 \%$ (major), 93.6\% ee

For 1f<smiles>C[As](C)(C)c1ccc(Cl)cc1</smiles>

$(+)-1 f$

For 1 g<smiles>C[S+](C)(O)c1ccc(F)cc1</smiles>

$(+)-1 g$
HPLC (CHIRALPAK IH, acetonitrile, flow rate $=0.5$ $\mathrm{mL} / \mathrm{min}, 1=254 \mathrm{~nm}$ ) $\mathrm{tR}=14.3 \mathrm{~min}, 99.6 \%$ (major), $16.4 \mathrm{~min}, 0.4 \%$ (minor), $99.2 \%$ ee
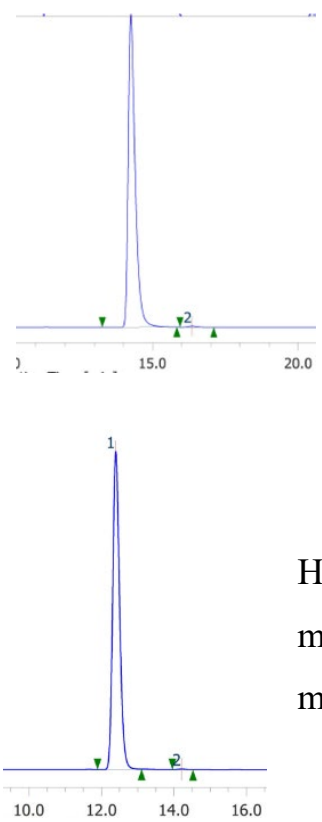

HPLC (CHIRALPAK IH, acetonitrile, flow rate $=0.5$ $\mathrm{mL} / \mathrm{min}, 1=254 \mathrm{~nm}$ ) $\mathrm{tR}=12.4 \mathrm{~min}, 99.8 \%$ (major), 14.2 $\min , 0.2 \%$ (minor), $99.6 \%$ ee

For $1 \mathbf{h}$<smiles>CO[13C](C)(C)c1cccc(F)c1</smiles>

$(+)-1 \mathrm{~h}$

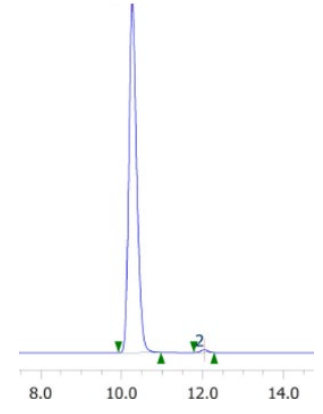

HPLC (CHIRALPAK IG, acetonitrile, flow rate = $0.5 \mathrm{~mL} / \mathrm{min}, 1=254 \mathrm{~nm}) \mathrm{tR}=10.3 \mathrm{~min}, 99.3 \%$ (major), $12.0 \mathrm{~min}, 0.7 \%$ (minor), $98.6 \%$ ee 
For 1i<smiles>C[As](C)(C)c1ccccc1F</smiles>

$(+)-1 \mathbf{i}$

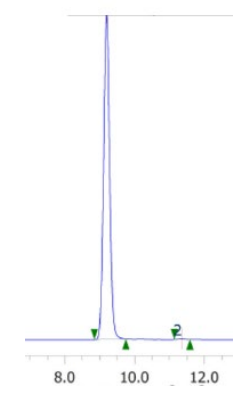

HPLC (CHIRALPAK IH, acetonitrile, flow rate $=0.5 \mathrm{~mL} / \mathrm{min}$, $1=254 \mathrm{~nm}$ ) $\mathrm{tR}=9.2 \mathrm{~min}, 99.8 \%$ (major), $11.4 \mathrm{~min}, 0.2 \%$ (minor), $99.6 \%$ ee

For $\mathbf{1 j}$<smiles>C[As](C)(O)c1ccccn1</smiles>

$(+)-\mathbf{1} \mathbf{j}$

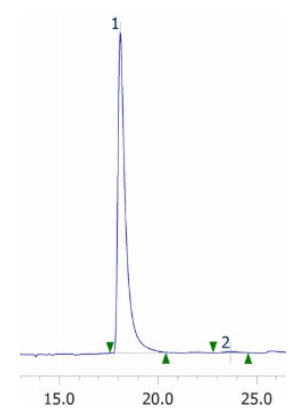

HPLC (CHIRALPAK IH, acetonitrile, flow rate $=0.5$ $\mathrm{mL} / \mathrm{min}, 1=254 \mathrm{~nm}$ ) $\mathrm{tR}=18.1 \mathrm{~min}, 99.5 \%$ (major), 23.7 min, $0.5 \%$ (minor), $99.0 \%$ ee

For 1k<smiles>CC[S+](C)(=O)c1ccccc1</smiles>

$(+)-1 k$

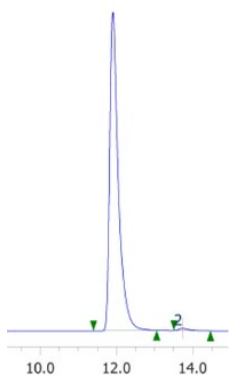

HPLC (CHIRALPAK IH, acetonitrile, flow rate $=0.5$ $\mathrm{mL} / \mathrm{min}, 1=254 \mathrm{~nm}$ ) $\mathrm{tR}=11.9 \mathrm{~min}, 99.3 \%$ (major), 13.7 $\min , 0.7 \%$ (minor), $98.6 \%$ ee

For 11<smiles>C[S+]([O-])(c1ccccc1)C1CC1</smiles>

HPLC (CHIRALPAK IH, acetonitrile, flow rate = $0.5 \mathrm{~mL} / \mathrm{min}, 1=254 \mathrm{~nm}) \mathrm{tR}=11.3 \mathrm{~min}, 96.6 \%$ (major), $16.4 \mathrm{~min}, 3.4 \%$ (minor), $93.2 \%$ ee

$(+)-11$

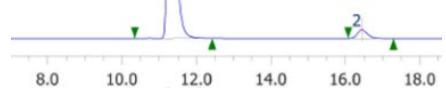


For $1 \mathbf{m}$<smiles>C[S+]([O-])(CCCl)c1ccccc1</smiles>

$(+)-1 \mathrm{~m}$

For 1n<smiles>C[Si](CCO)(CCO)c1ccccc1</smiles>

$(+)-1 n$

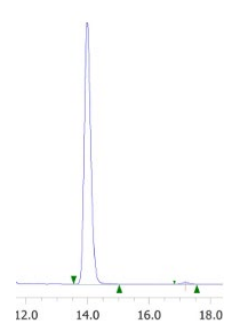

HPLC (CHIRALPAK IG, acetonitrile, flow rate $=0.5$ $\mathrm{mL} / \mathrm{min}, 1=254 \mathrm{~nm}$ ) $\mathrm{tR}=14.0 \mathrm{~min}, 99.3 \%$ (major), 17.2 $\min , 0.7 \%$ (minor), $98.6 \%$ ee

For 10<smiles>COCC[S+](C)(=O)c1ccccc1</smiles>

$(+)-10$

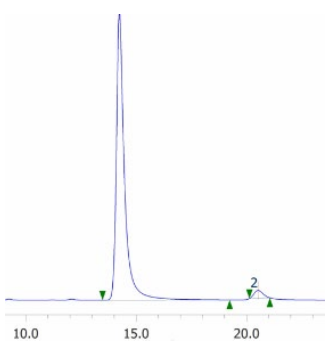

HPLC (CHIRALPAK IF, acetonitrile, flow rate $=$ $0.5 \mathrm{~mL} / \mathrm{min}, 1=254 \mathrm{~nm}) \mathrm{tR}=14.2 \mathrm{~min}, 97.1 \%$ (major), 20.5 min, 2.8\% (minor), 94.3\% ee

HPLC (CHIRALPAK ID, acetonitrile, flow rate $=0.5 \mathrm{~mL} / \mathrm{min}, 1=254 \mathrm{~nm}) \mathrm{tR}=9.9 \mathrm{~min}, 96.1 \%$ (major), $12.8 \mathrm{~min}, 3.8 \%$ (minor), $92.3 \%$ ee

For 1p<smiles>CCOC(=O)C[S+](C)(=O)c1ccccc1</smiles>
$(+)-1 p$

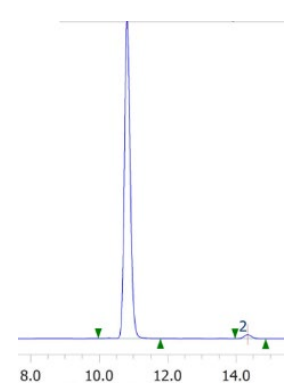

HPLC (CHIRALPAK IG, acetonitrile, flow rate $=0.5$ $\mathrm{mL} / \mathrm{min}, 1=254 \mathrm{~nm}$ ) $\mathrm{tR}=10.8 \mathrm{~min}, 98.8 \%$ (major), $14.3 \mathrm{~min}, 1.2 \%$ (minor), $97.6 \%$ ee 
For 1q<smiles>COC(=O)CC[S+](C)(=O)c1ccccc1</smiles>

$(+)-1 q$

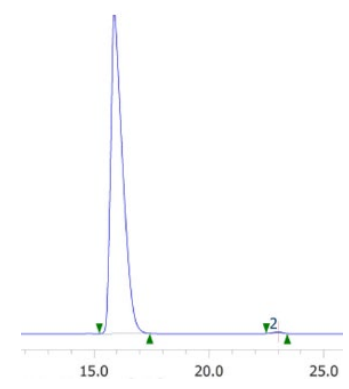

HPLC (CHIRALPAK IF, acetonitrile, flow rate $=0.5 \mathrm{~mL} / \mathrm{min}, 1=254 \mathrm{~nm}) \mathrm{tR}=15.9 \mathrm{~min}$, 99.6\% (major), $23.0 \mathrm{~min}, 0.4 \%$ (minor), $99.2 \%$ ee

For 1r<smiles>CC(=O)OCC[S+](C)(C)c1ccccc1</smiles>

$(+)-1 \mathbf{r}$
HPLC (CHIRALPAK IF, acetonitrile, flow rate $=0.5$ $\mathrm{mL} / \mathrm{min}, 1=254 \mathrm{~nm}$ ) $\mathrm{tR}=12.3 \mathrm{~min}, 1.6 \%$ (minor), 14.7 min, $98.4 \%$ (major), $96.8 \%$ ee

For $1 \mathrm{~s}$<smiles>Cc1ccc([S+](C)(O)c2ccccc2)cc1</smiles>

(+)-1s

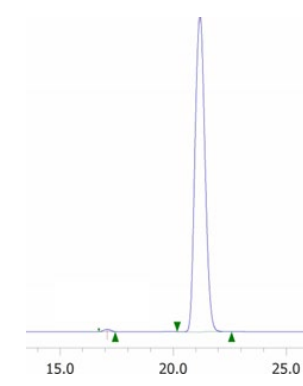

HPLC (CHIRALPAK IG, acetonitrile, flow rate $=$ $0.5 \mathrm{~mL} / \mathrm{min}, 1=254 \mathrm{~nm}) \mathrm{tR}=17.1 \mathrm{~min}, 0.5 \%$ (minor), $21.2 \mathrm{~min}, 99.5 \%$ (major), 99.0\% ee

For 1t<smiles>C=C[Si](C)(O)c1ccccc1</smiles>

1t

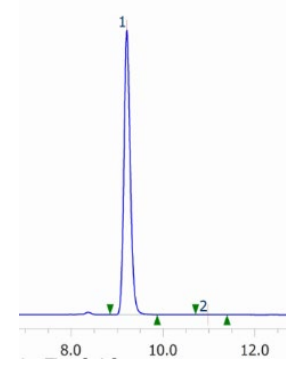

HPLC (CHIRALPAK IG, acetonitrile, flow rate $=0.5$ $\mathrm{mL} / \mathrm{min}, 1=254 \mathrm{~nm}$ ) $\mathrm{tR}=9.2 \mathrm{~min}, 99.9 \%$ (major minor), 10.9 min, $0.1 \%$ (minor), $99.8 \%$ ee 
For $1 \mathbf{u}$

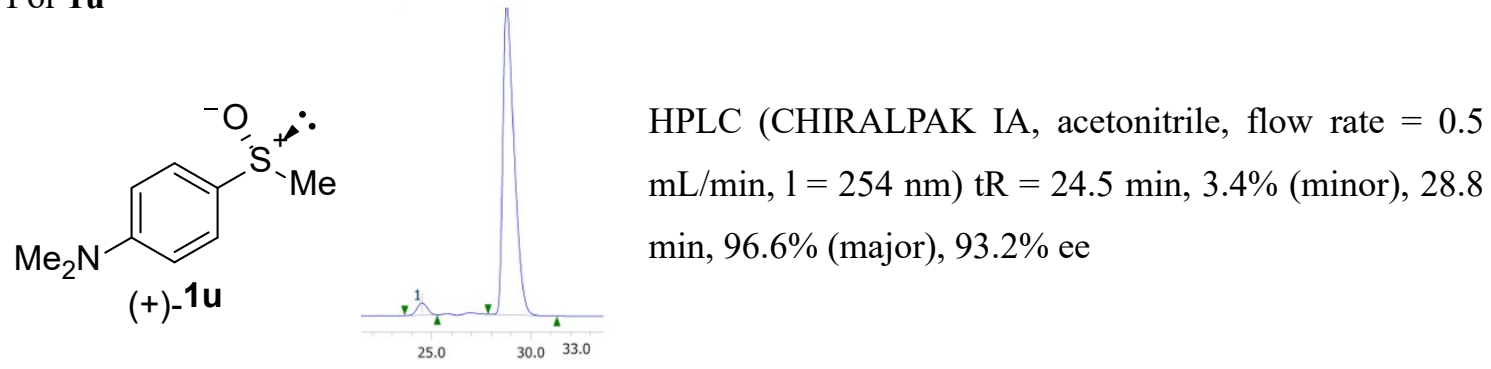

For 1v<smiles>[3H][S@@](O)(CCN1CCCC1)c1ccccc1</smiles>

$(+)-1 v$

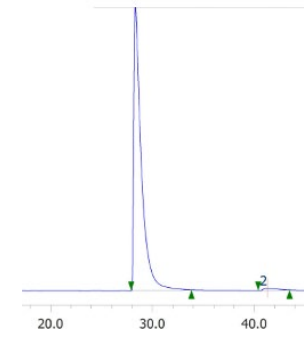

HPLC (CHIRALPAK IG, acetonitrile, flow rate = $0.5 \mathrm{~mL} / \mathrm{min}, 1=254 \mathrm{~nm}) \mathrm{tR}=28.3 \mathrm{~min}, 98.7 \%$ (major), $41.3 \mathrm{~min}, 1.3 \%$ (minor), 97.4\% ee

For 1w<smiles></smiles>

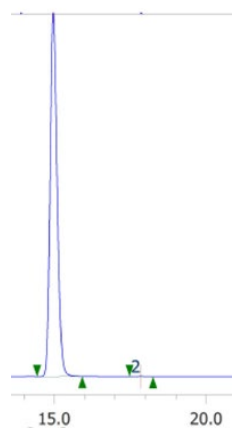

HPLC (CHIRALPAK IA, acetonitrile, flow rate $=0.5 \mathrm{~mL} / \mathrm{min}, 1=254 \mathrm{~nm}) \mathrm{tR}=15.0 \mathrm{~min}$, 99.9\% (major), $17.8 \mathrm{~min}, 0.1 \%$ (minor), $99.8 \%$ ee 
7. ${ }^{1} \mathrm{H}-,{ }^{13} \mathrm{C}$-, and $2 \mathrm{D}-\mathrm{NMR}$ spectra of $1 \mathrm{v}, 2,1 \mathrm{w}$.

${ }^{1} \mathrm{H}-\mathrm{NMR}\left(400 \mathrm{MHz}, \mathrm{CDCl}_{3}\right.$ ) of $\mathbf{1 v}$

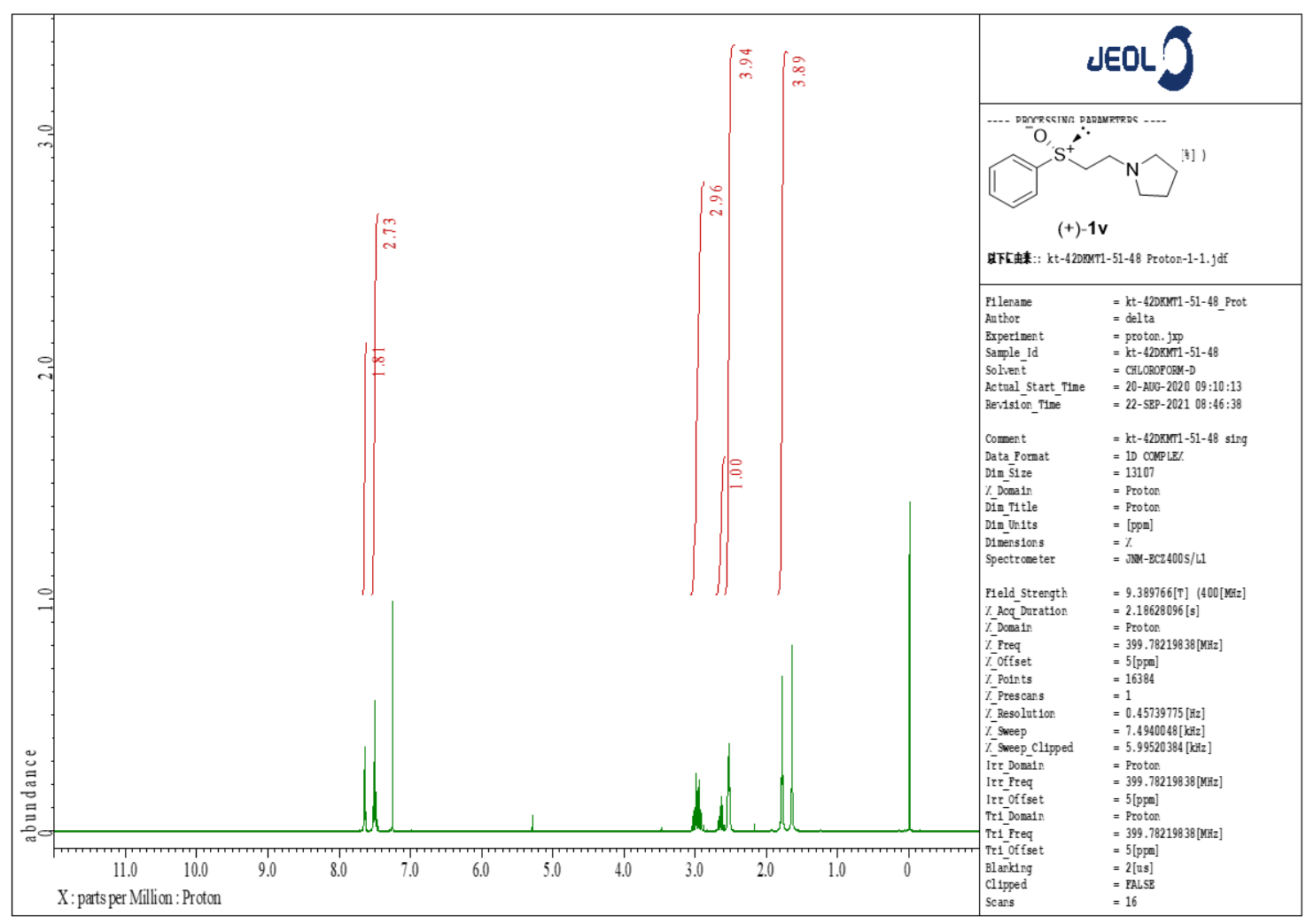

${ }^{13} \mathrm{C}\left\{{ }^{1} \mathrm{H}\right\}-\mathrm{NMR}\left(100 \mathrm{MHz}, \mathrm{CDCl}_{3}\right)$ of $\mathbf{1 v}$

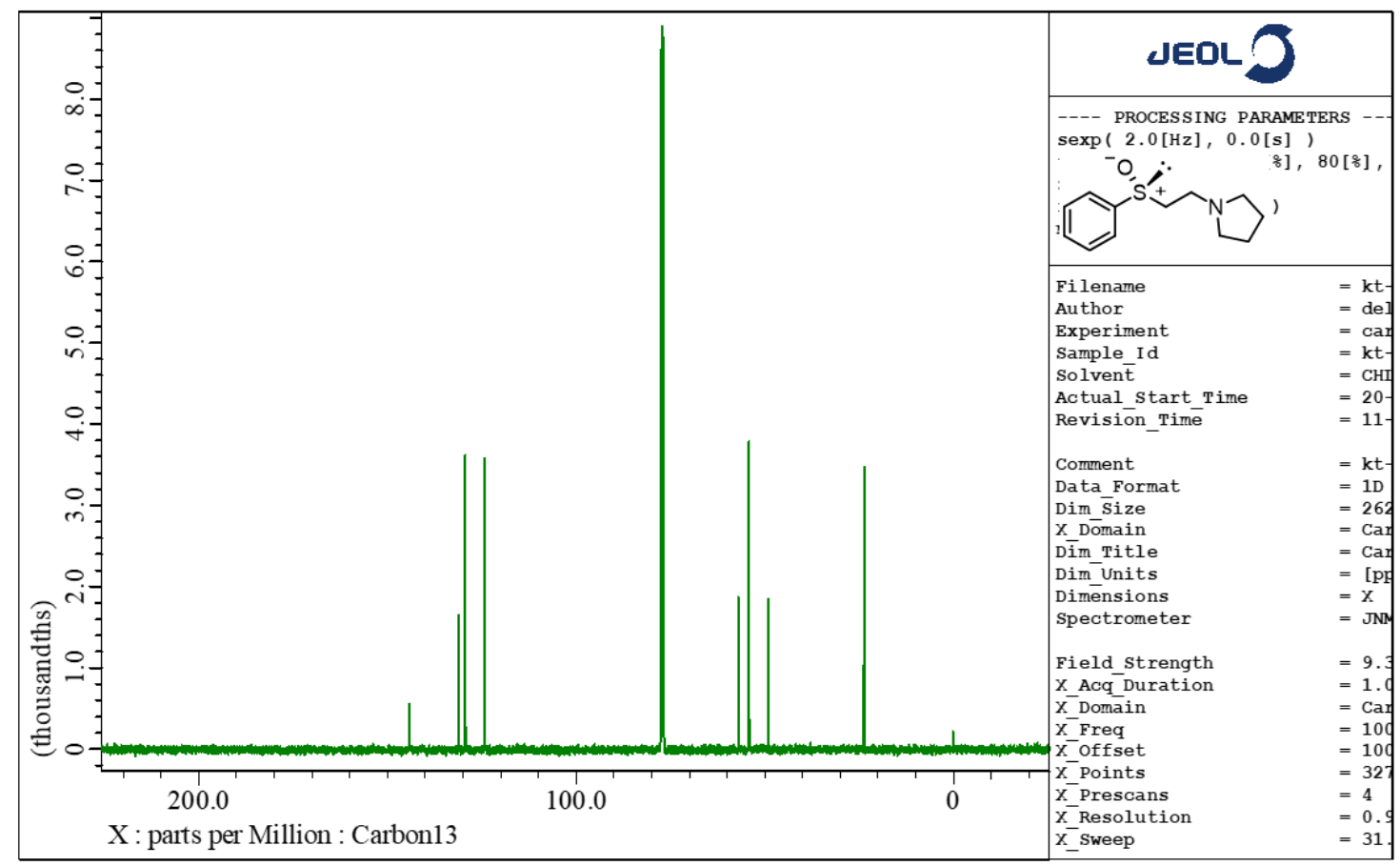


H-H COSY-NMR (400 MHz, $\mathrm{CDCl}_{3}$ ) of $\mathbf{1 v}$

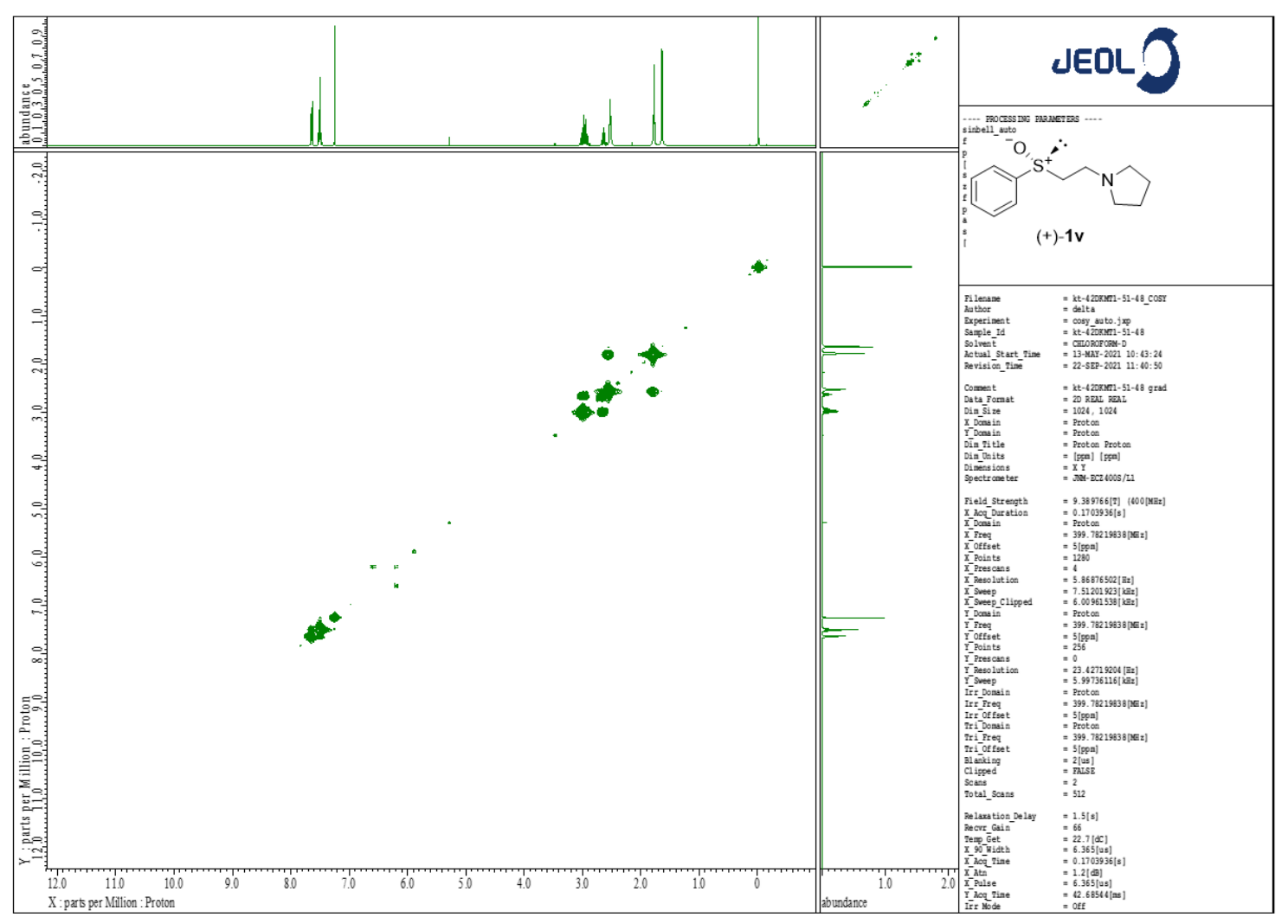

HMQC-NMR $\left(\mathrm{CDCl}_{3}\right)$ of $\mathbf{1 v}$

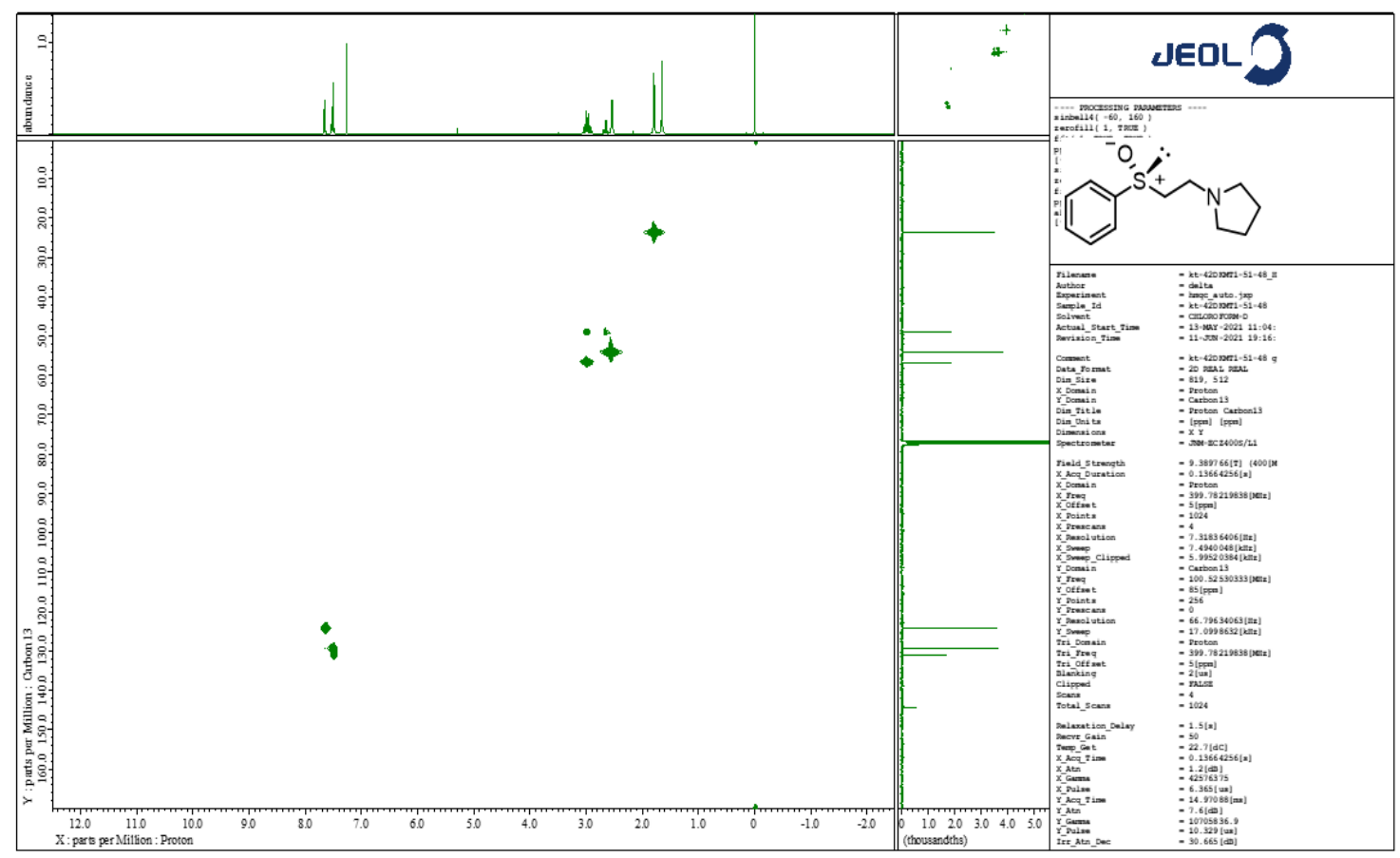


${ }^{1} \mathrm{H}-\mathrm{NMR}\left(400 \mathrm{MHz}, \mathrm{CDCl}_{3}\right.$ ) of 2

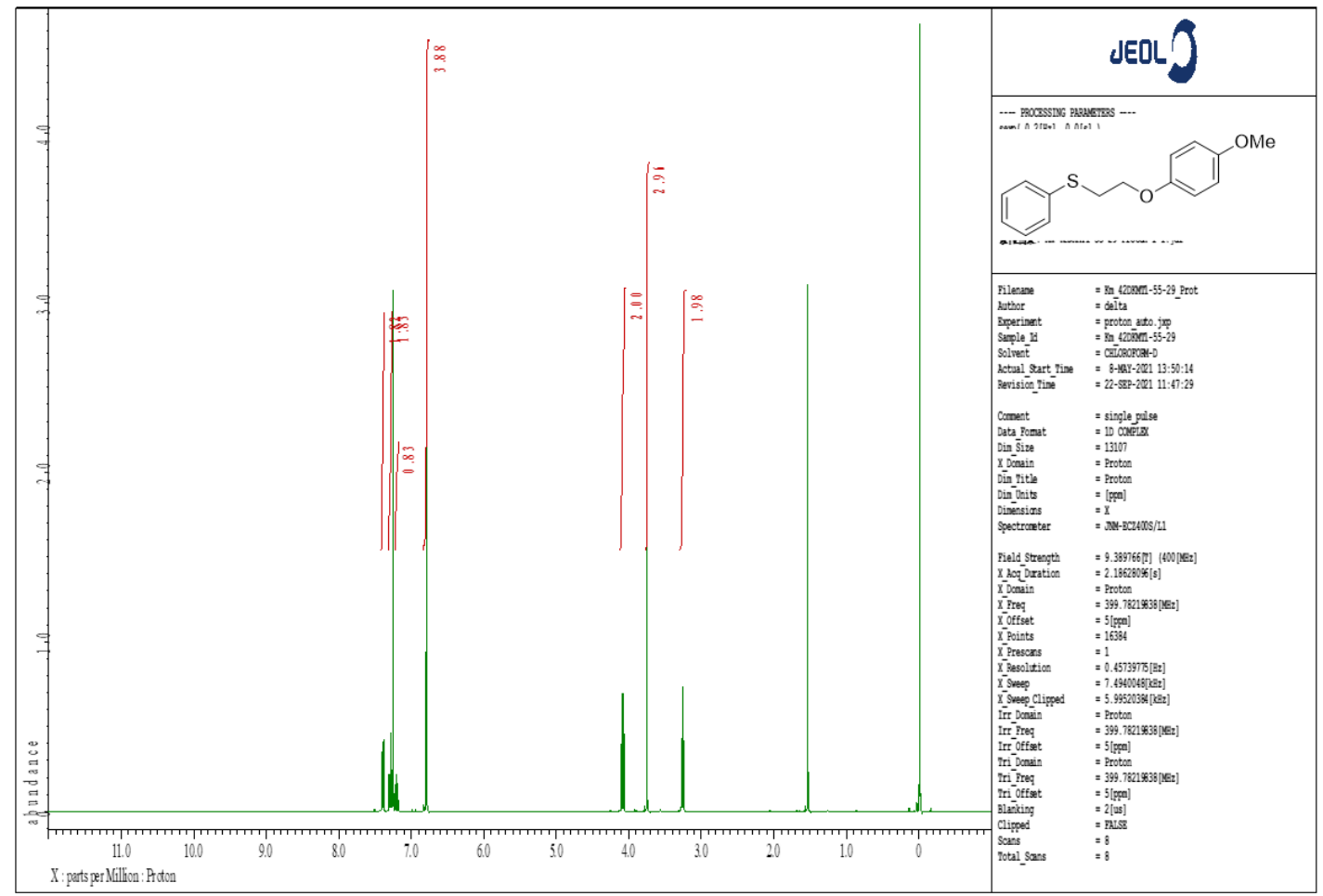

${ }^{13} \mathrm{C}\left\{{ }^{1} \mathrm{H}\right\}$-NMR $\left(100 \mathrm{MHz}, \mathrm{CDCl}_{3}\right)$ of 2

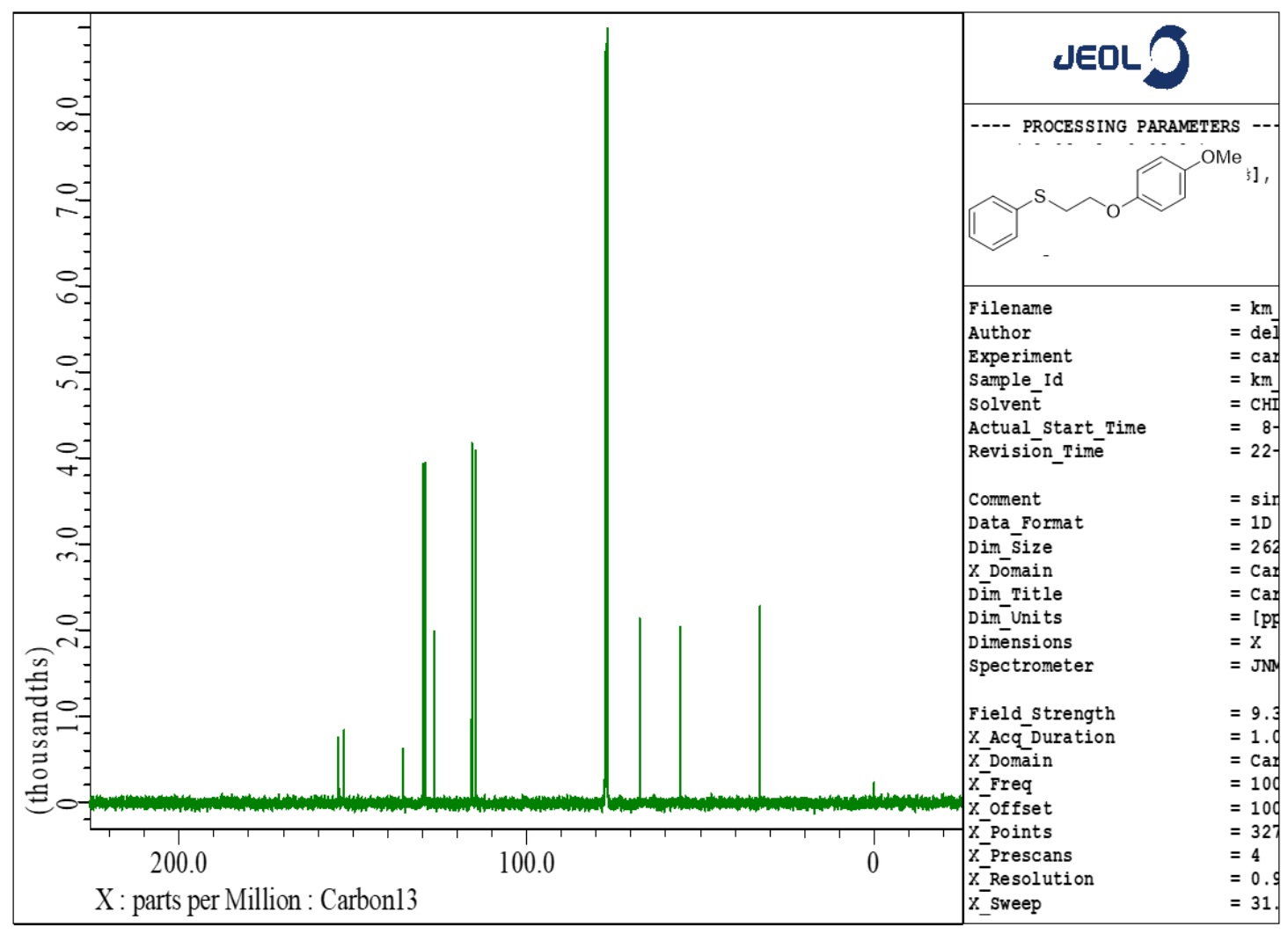


H-H COSY-NMR (400 MHz, $\left.\mathrm{CDCl}_{3}\right)$ of 2

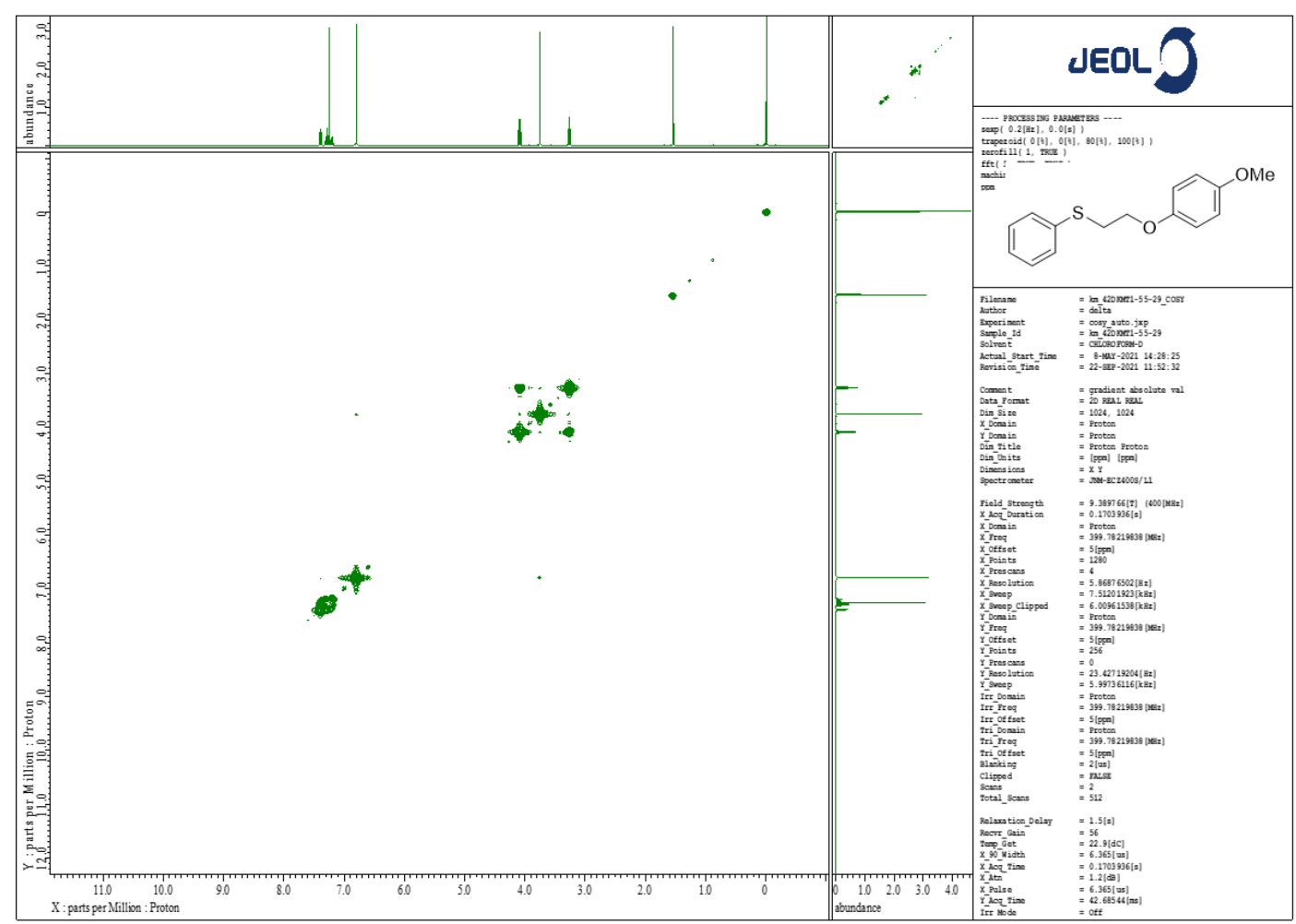

HMQC-NMR $\left(\mathrm{CDCl}_{3}\right)$ of 2

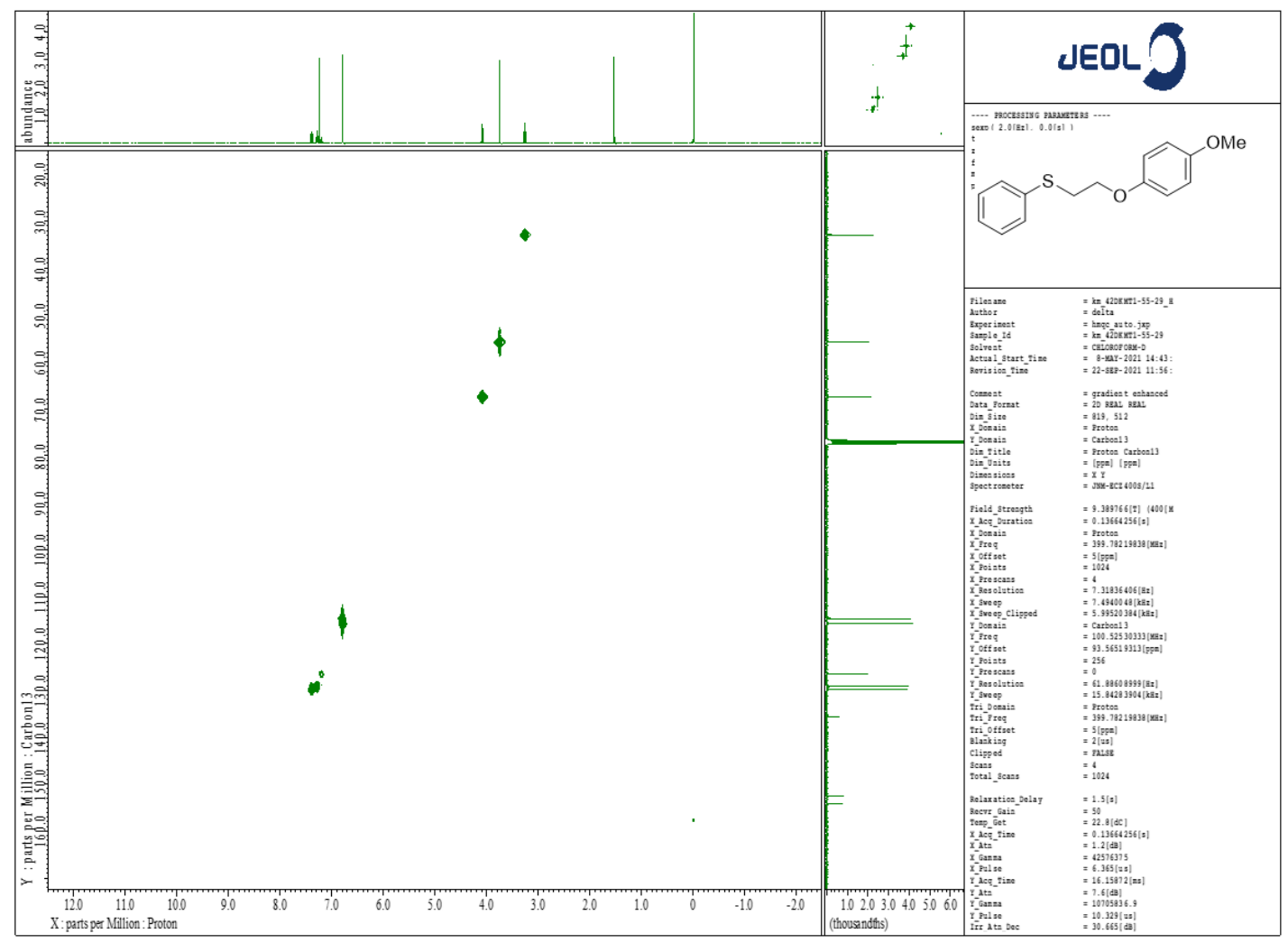


${ }^{1} \mathrm{H}-\mathrm{NMR}\left(400 \mathrm{MHz}, \mathrm{CDCl}_{3}\right.$ ) of $\mathbf{1 w}$

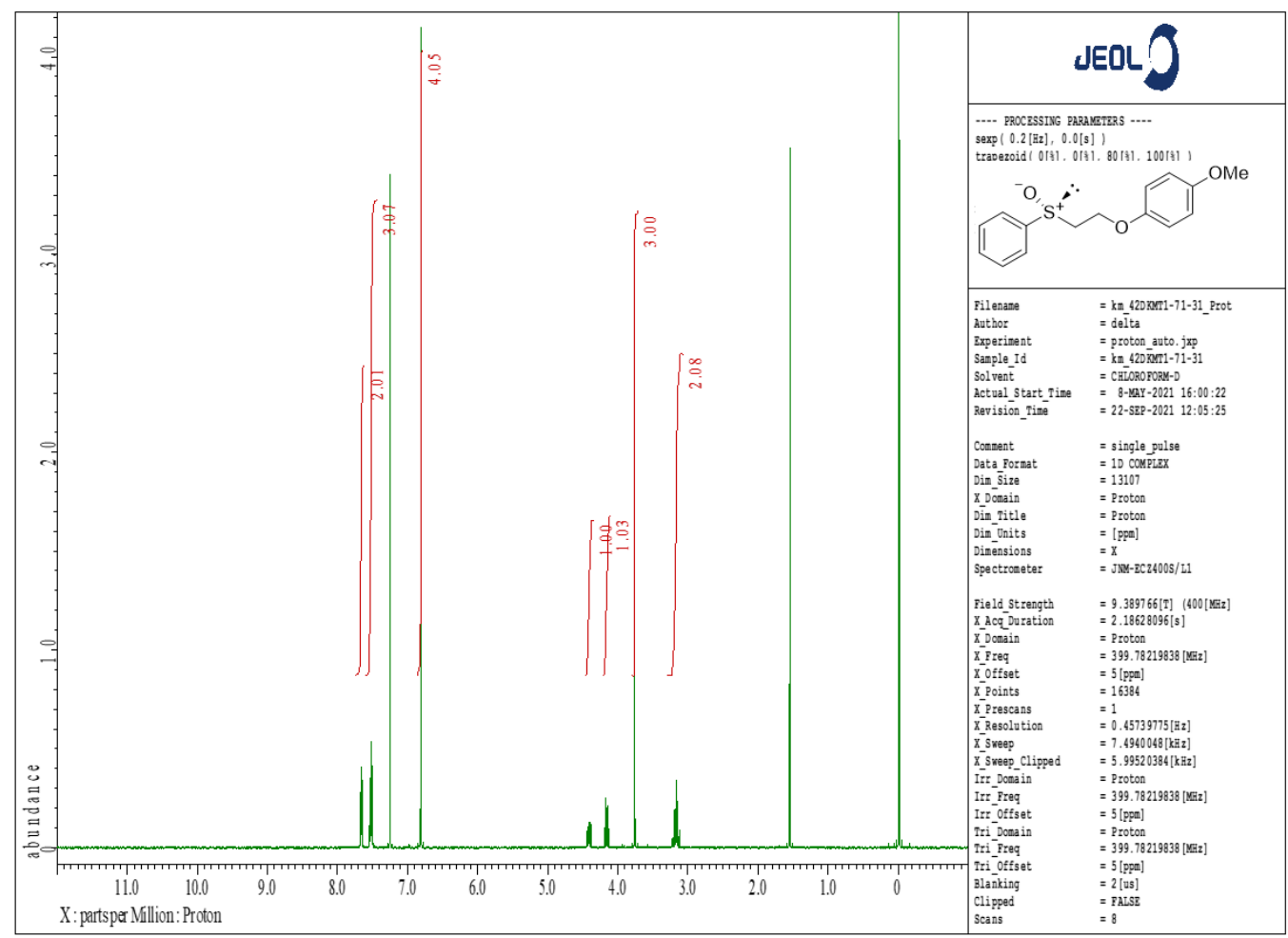

${ }^{13} \mathrm{C}\left\{{ }^{1} \mathrm{H}\right\}-\mathrm{NMR}\left(100 \mathrm{MHz}, \mathrm{CDCl}_{3}\right)$ of $\mathbf{1 w}$

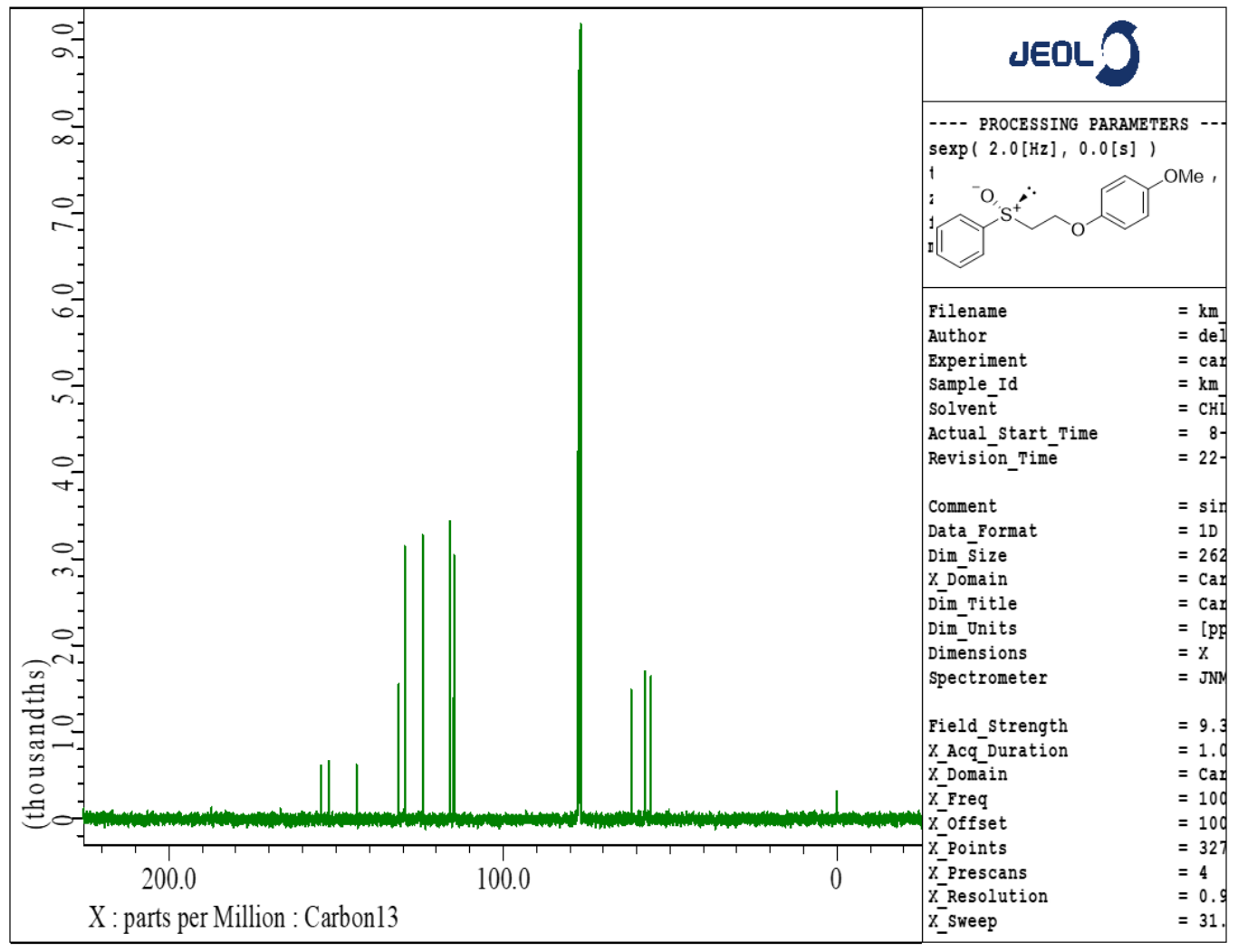


H-H COSY-NMR (400 MHz, $\left.\mathrm{CDCl}_{3}\right)$ of $\mathbf{1} \mathbf{w}$

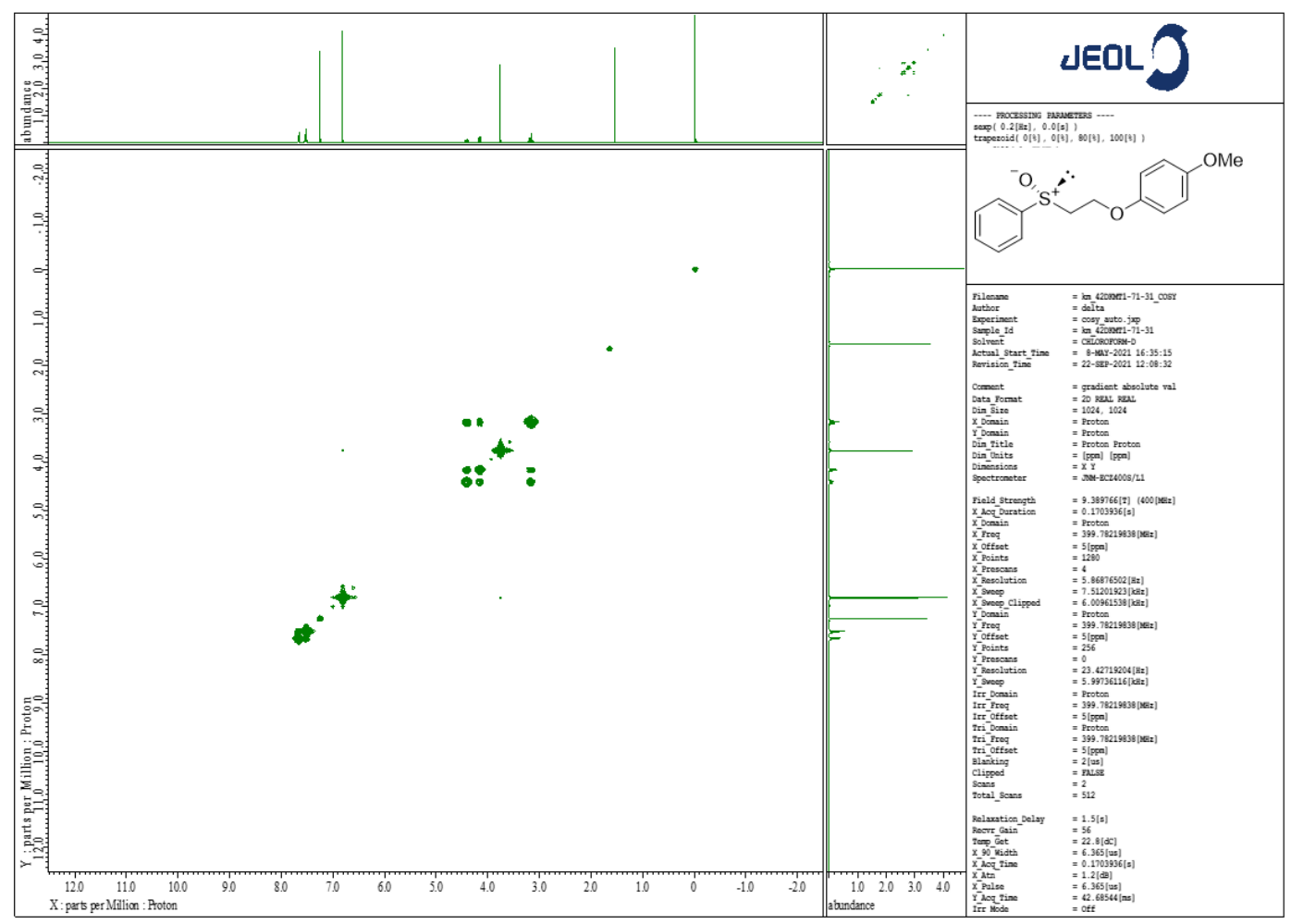

HMQC-NMR $\left(\mathrm{CDCl}_{3}\right)$ of $\mathbf{1 w}$

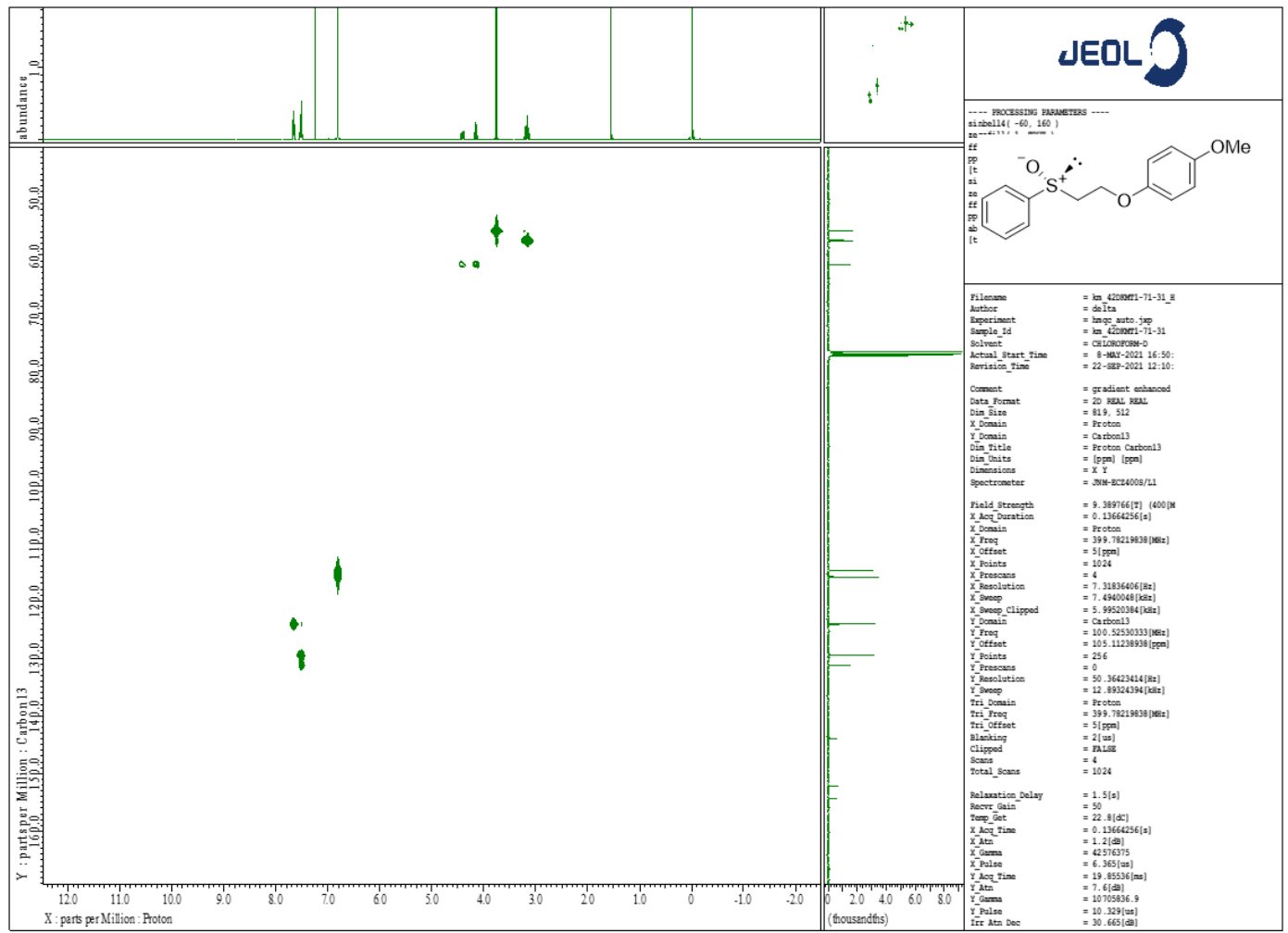



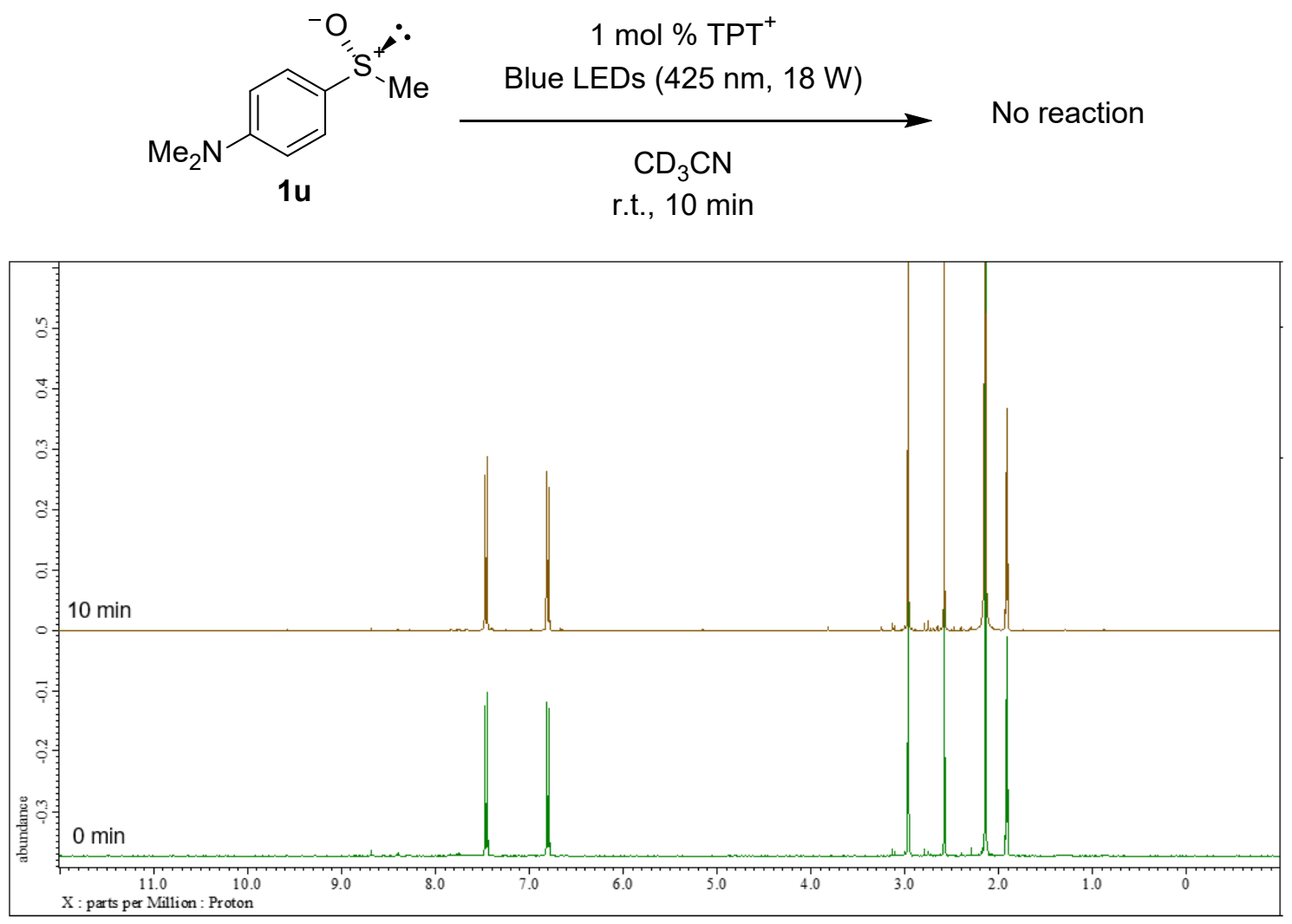


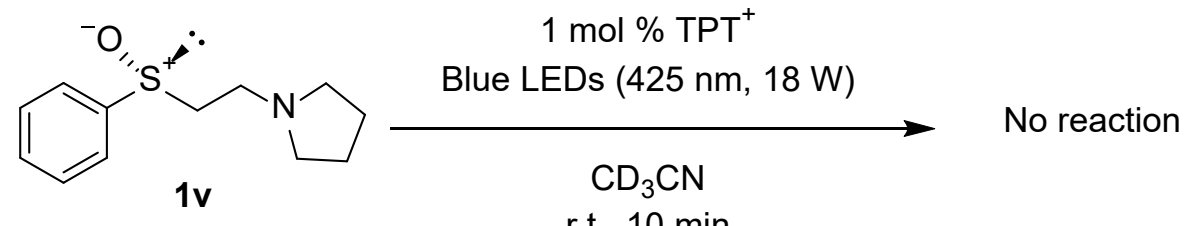

r.t., $10 \mathrm{~min}$

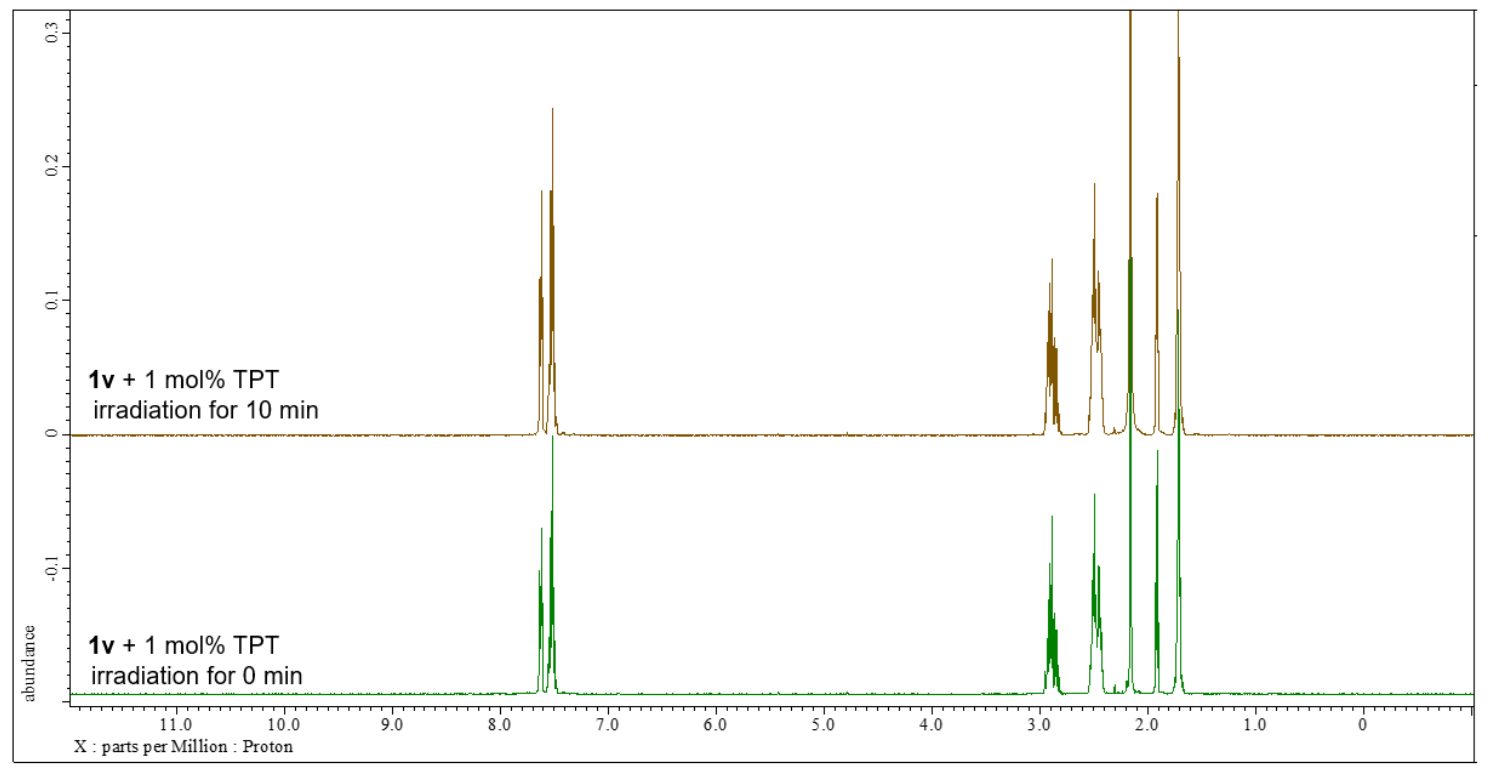



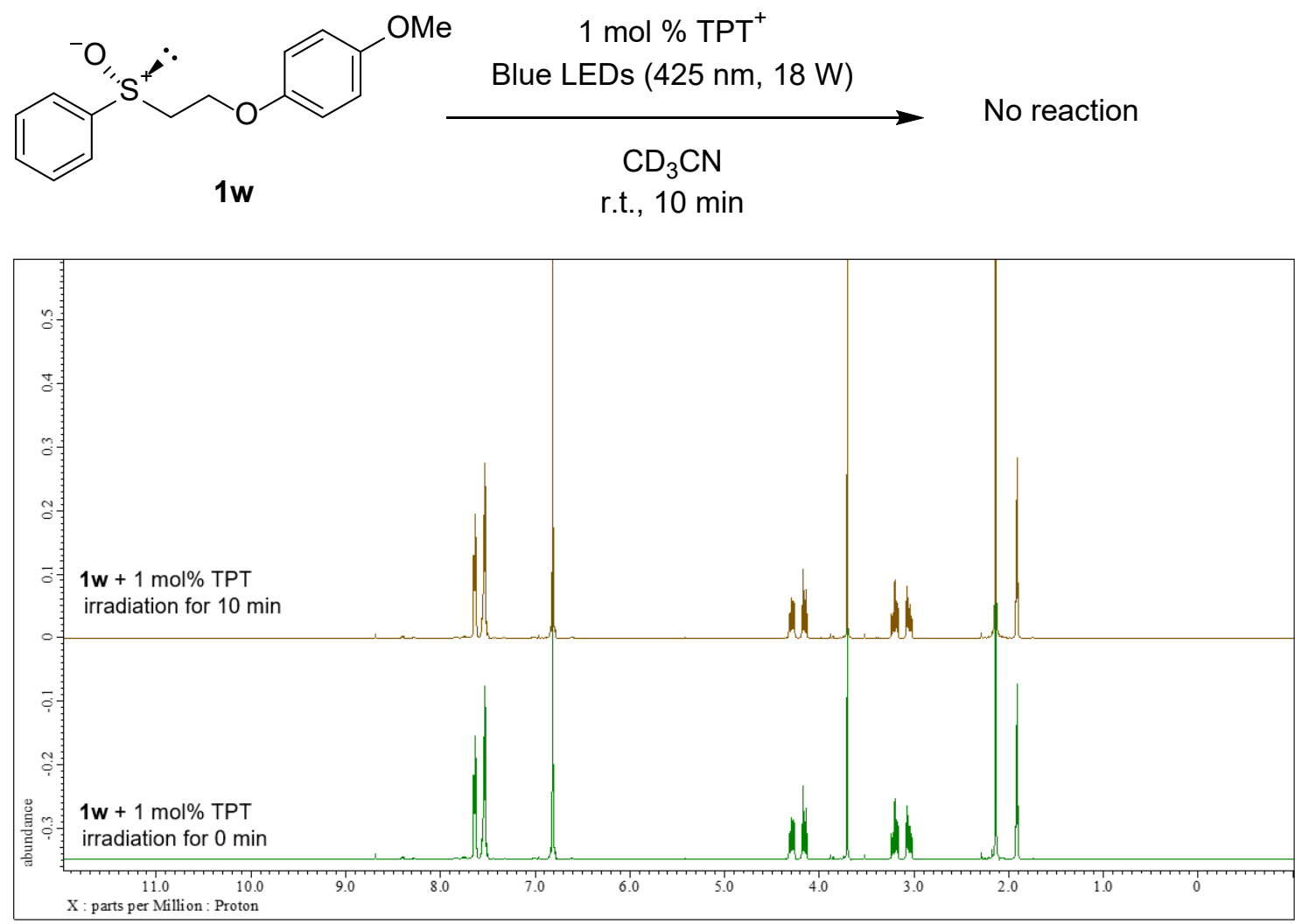